\title{
Saddle-center and periodic orbit: dynamics near symmetric heteroclinic connection
}

\author{
L.M. Lerman ${ }^{a, b}$, K.N. Trifonov ${ }^{b}$ \\ ${ }^{a}$ National Research University Higher School of Economics, \\ ${ }^{b}$ Scientific and Educational Mathematical Center "Mathematics of Future Technologies" \\ Lobachevsky State Research University of Nizhny Novgorod, Russia
}

\begin{abstract}
An analytic reversible Hamiltonian system with two degrees of freedom is studied in a neighborhood of its symmetric heteroclinic connection made up of a symmetric saddle-center, a symmetric orientable saddle periodic orbit lying in the same level of a Hamiltonian and two non-symmetric heteroclinic orbits permuted by the involution. This is a codimension one structure and therefore it can be met generally in one-parameter families of reversible Hamiltonian systems. There exist two possible types of such connections in dependence on how the involution acts near the equilibrium. We prove a series of theorems which show a chaotic behavior of the system and those in its unfoldings, in particular, the existence of countable sets of transverse homoclinic orbits to the saddle periodic orbit in the critical level, transverse heteroclinic connections involving a pair of saddle periodic orbits, families of elliptic periodic orbits, homoclinic tangencies, families of homoclinic orbits to saddle-centers in the unfolding, etc. As a byproduct, we get a criterion of the existence of homoclinic orbits to a saddle-center.
\end{abstract}

The orbit structure of a non-integrable Hamiltonian system with two or more degrees of freedom is very complicated and usually it is impossible, except for some specific model situations, to describe its structure more or less completely. By this reason, a fruitful way to understand the orbit behavior in some parts of the phase space is to detect some simple invariant subsets (usually containing a finite number of orbits) whose neighborhoods can be understood from the viewpoint of their orbit structure. When it has been done, we try to find such structures in general systems and thus to describe partially the behavior of the system under study. This approach goes back to A. Poincaré. The problem investigated here follow these lines. It was inspired by the study of stationary waves in a nonlocal Whitham equation [25] that is reduced to the reversible Hamiltonian system with two degrees of freedom for which homoclinic orbits to different type of equilibria have to be detected. We rely in this research on earlier results on the behavior near a homoclinic orbit to a saddle-center equilibrium [19, 20, 27, 9, 8, 43] as well as near homoclinic tangencies [31, 28, [5, 3, 6, 15, 16]. The results obtained demonstrate how much can be understood at this approach.

\section{Contents}

1 Introduction 
2 Setting up and main notions 4

3 Moser coordinates 5

4 Local orbit structure near saddle-center

5 Poincaré map in a neighborhood of $\gamma \quad 8$

6 Global maps

7 Types of symmetric connections

8 In the singular level $V_{0}$

9 Hyperbolicity and ellipticity in levels $c<0$

10 Hyperbolicity and ellipticity as $c>0$

11 The case $2, c>0$

12 1-parameter family of reversible systems:
homoclinics of the saddle-center

13 Conclusion

14 Acknowledgement 27

15 Data availability 


\section{Introduction}

Studying Hamiltonian dynamics is an interesting and hard problem attracting researchers from many branches of science since Hamiltonian systems serve as mathematical models in different problems in physics, chemistry and engineering. The structure of such systems is usually rather complicated, therefore one of a fruitful approach to these type of problems is the study of the given system near some invariant sets which can be selected by simple conditions. Studying systems in neighborhoods of homoclinic orbits and heteroclinic connections is the problem of such type. Investigations of dynamical phenomena near a homoclinic orbit to a saddle periodic orbit was the first such problem, its set up and understanding the complexity of orbit behavior of the system near such structure goes back to Poincaré [33]. The real complexity of orbit behavior was understood due to works by Birkhoff [1], Smale [40] and finally Shilnikov [35]. Other problems, where complicated dynamics was detected, were studied in many papers by Shilnikov and coauthors, among them the most influential are [36, 39, 4, 37]. Homoclinic dynamics in Hamiltonian systems began studying in [12] where Shilnikov results about the complicated dynamics near a saddle-focus homoclinic loop were carried over to Hamiltonian case. The generalization of the Melnikov method onto the autonomous case for systems close to Hamiltonian integrable [24] allowed one to present examples of a complicated behavior both for Hamiltonian perturbation of an integrable Hamiltonian system with a saddle-focus skirt and for dissipative perturbations. The complicated dynamics near a bunch of homoclinic orbits to a saddle in a Hamiltonian system was detected in [38] (see generalizations of these results in [42, 17]). More close to the topic of the present paper results of [22] are where was studied first a complicated dynamics near a saddle-center homoclinic loop, when the equilibrium was not hyperbolic. The set-up for this problem was earlier presented in [2], but no essential results were found there. Results of [22] was later extended in different directions in [19, 20, 27, 9, 8, 43].

In this paper the dynamics is studied in a reversible Hamiltonian system with two degrees of freedom in a neighborhood of a symmetric heteroclinic connection which consists of a symmetric saddle-center, a symmetric saddle periodic orbit which are connected by two nonsymmetric heteroclinic orbits being permuted by the involution. (see. Fig,1).

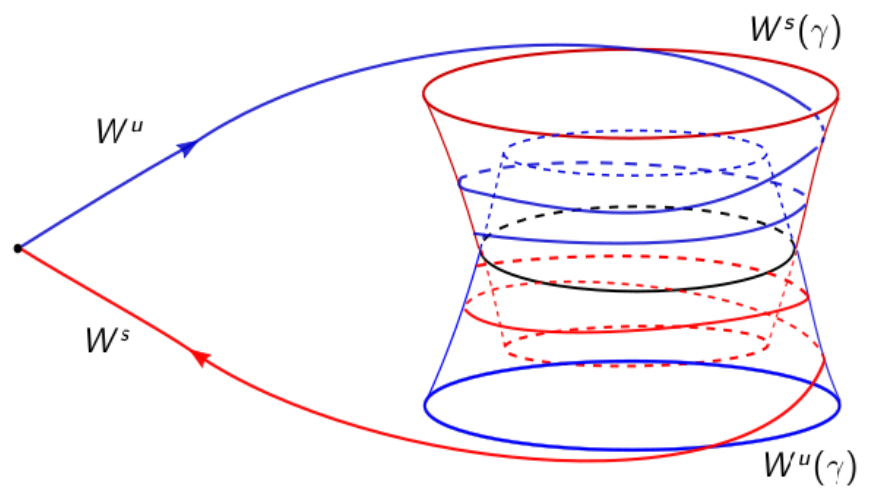

Figure 1: Scheme of the heteroclinic connection

This type of connection is a codimension one phenomenon in the class of reversible Hamiltonian systems. Thus, such a structure can irreparably appear in one-parameter families. Systems with such structures are met in applications. For instance, they were discovered in [25] where 
solitons in a nonlocal Whitham equation [26] were studied. Also this structure can be found in a one parameter family of reversible Hamiltonian systems near a destruction of a homoclinic orbit to a saddle-center. Results of [19] suggest that saddle periodic orbits accumulate to the saddlecenter loop in the singular level of the Hamiltonian and therefore, after destruction, unstable separatrix of the symmetric saddle-center can lie on the stable manifold of a symmetric saddle periodic orbit. Due to reversibility, there is a pairing stable separatrix of the saddle-center that lie on the unstable manifold of the same periodic orbit as it is symmetric.

One more application of results obtained is a criterion of the existence of saddle-center homoclinic loops in a reversible Hamiltonian system (Theorem 11 below).

Main results of the paper prove the existence of hyperbolic sets and elliptic periodic orbits near the heteroclinic connection. Existence of hyperbolic sets is based on the construction of families of transverse homoclinic orbits and heteroclinic connections involving two saddle periodic orbits and four transverse heteroclinic orbits for them. Existence of elliptic periodic orbits is prove by the same scheme. We look for homoclinic orbits with quadratic tangencies to saddle periodic orbits in different situations and apply then results of going back to Newhouse [31, 32] and Gavrilov-Shilnikov [4] and many others [31, 28, [5, 3, 6, 15, 16] on the existence of cascades of elliptic periodic orbits.

\section{Setting up and main notions}

Let $(M, \Omega)$ be a real analytic four-dimensional symplectic manifold, $\Omega$ be its symplectic 2 -form and $H$ be a real analytic function (a Hamiltonian). Such function defines a Hamiltonian vector field $X_{H}$ on $M$. Henceforth we assume $X_{H}$ to have an equilibrium $p$ of the saddle-center type and without a loss of generality we assume $H(p)=0$. One more assumption we use is the existence in the same level of $H=0$ a saddle periodic orbit $\gamma$. We use below the notation $V_{c}=\{x \in M \mid H(x)=c\}$.

Recall an equilibrium $p$ of $X_{H}$ on $M$ is called to be a saddle-center [22], if the linearization operator of the vector field at $p$ has a pair of pure imaginary eigenvalues $\pm i \omega, \omega \in \mathbb{R} \backslash\{0\}$, and a pair nonzero reals $\pm \lambda \neq 0$. In a neighborhood of such equilibrium the system has a unique local invariant smooth two-dimensional invariant symplectic submanifold $W_{p}^{c}$ filled with closed orbits $l_{c}$ (Lyapunov family of periodic orbits). For the case of analytic $M, H$ the submanifold $W_{p}^{c}$ is real analytic. Near the point $p$ in the level $H=c$ periodic orbit $l_{c}$ is of saddle type and is located each on its own level $V_{c}$.

Also in a neighborhood of $p$ the system has local 3-dimensional center-stable $W^{c s}$ and centerunstable manifold $W^{c u}$ containing both $p$. These submanifolds contain orbits being asymptotic, as $t \rightarrow \infty$ (for $W^{c s}$ ), to periodic orbits $l_{c}$, or as $t \rightarrow-\infty$ (for $W^{c u}$ ), here $W^{c}=W^{c s} \cap W^{c u}$. Submanifold $W^{c s}$ (respectively, $W^{c u}$ ) is foliated by levels $V_{c}$ into local stable (unstable) manifolds of periodic orbits $l_{c}$, these submanifolds are diffeomorphic to cylinders $I \times S^{1}$. Besides, $W^{c s}$ (respectively, $W^{c u}$ ), being a solid cylinder, contains as an axis, an analytic curve $W^{s}\left(W^{u}\right)$ - local stable (unstable) manifold of the equilibrium $p$, they consist of $p$ and two semi-orbits tending $p$ as $t \rightarrow \infty(t \rightarrow-\infty)$.

As was supposed above, $V_{0}$ contains a saddle periodic orbit $\gamma$. In the whole $M$ orbit $\gamma$ belongs to a one-parameter family of such periodic orbits $\gamma_{c} \subset V_{c}$ forming an analytic 2-dimensional symplectic cylinder. Recall that in $V_{c}$ periodic orbit $\gamma_{c}$ has two local analytic 2-dimensional Lagrangian submanifolds $W^{s}(\gamma), W^{u}(\gamma)$ being its stable and unstable local submanifolds. All 
of them are topologically either cylinders (if its multipliers are positive) or Möbius strips (if its multipliers are negative).

Later on in the paper the vector field $v=X_{H}$ under consideration is supposed to be reversible as well. This means [13] that on $M$ acts a smooth involution $L: M \rightarrow M, L^{2}=i d_{M}$, и $v$ obeys the identity $D L(v)=-v \circ L$. For its solutions this property reads as follows: if $x(t)$ is a solution to $v$, then $x_{1}(t)=L x(-t)$ is also its solution.

Orbit $\gamma$ of a reversible $v$ is called symmetric, if it is invariant w.r.t. the action of $L$. In particular, an equilibrium $p, v(p)=0$, is symmetric, if $L(p)=p$, i.e. $p$ belongs to the fixed point set of $L, F i x(L)=\{x \in M \mid L(x)=x\}$. The following statement holds true [13].

Proposition 1. An orbit of a reversible vector field is symmetric, iff it intersects Fix $(L)$. A symmetric periodic orbit intersects Fix $(L)$ at two points exactly. The inverse statement is also valid: if an orbit of a reversible vector field intersects Fix $(L)$ at two different points $x_{1}, x_{2}$, then this orbit is symmetric periodic one and its period is equal to the doubled transition time from $x_{1}$ to $x_{2}$.

For a Hamiltonian system the reversibility requires of a clarification, since the involution acts on the symplectic form

$$
\left[L^{*} \Omega\right](\xi, \eta)=\Omega(D L(\xi), D L(\eta)) .
$$

We assume below that an analytic involutive diffeomorphism $L$ is anti-canonical mapping, i.e. $L^{*}(\Omega)=-\Omega$ and $L$ is concordant with $H: H \circ L=H$. In this case the following identities hold:

$$
D L\left(X_{H}\right)=-X_{H} \circ L, \text { и } \Phi_{t} \circ L=L \circ \Phi_{-t} .
$$

We also assume $F i x(L)$ to be an analytic two-dimensional submanifold in $M$ (not obligatory connected).

Finally, we assume $X_{H}$ to have a heteroclinic connection consisting of a symmetric saddlecenter $p, H(p)=0$, a symmetric periodic orbit $\gamma$ in the same level of $H(\gamma)=0$ and two heteroclinic orbits: $\Gamma_{1}$ going, as $t$ increases, from $\gamma$ to $p$, and $\Gamma_{2}=L\left(\Gamma_{1}\right)$ going, as $t$ increases, from $p$ to $\gamma$. Our task is to study the orbit behavior in a neighborhood of this heteroclinic connection. It is worth observing that the problem, by its set up, is bifurcation, since the orbit structure varies as a values of the Hamiltonian $c$ varies near a critical value $c=0$. For instance, on the levels others than $V_{0}$, the equilibrium is absent thus the contour is destroyed and bifurcations are expected.

\section{Moser coordinates}

To examine orbit structure of the system near a connection, we shall use convenient coordinates in a neighborhood of $p$ and in a neighborhood of $\gamma$. Corresponding results in the analytic case are due to Moser [29, 30], a finite-smooth version for a saddle fixed point of a symplectic diffeomorphism exists in [15].

Theorem 1. Let $X_{H}$ be an analytic Hamiltonian vector field and $p$ its equilibrium of the saddlecenter type. Then there is a neighborhood $U$ of $p$, analytic symplectic coordinates $\left(x_{1}, y_{1}, x_{2}, y_{2}\right)$, $\Omega=d x_{1} \wedge d y_{1}+d x_{2} \wedge d y_{2}$, and a real analytic function $h(\xi, \eta)$, such that $H$ casts in the form

$$
H\left(x_{1}, y_{1}, x_{2}, y_{2}\right)=h(\xi, \eta)=\lambda \xi+\omega \eta+R(\xi, \eta), \quad R(\xi, \eta)=O\left(\xi^{2}+\eta^{2}\right), \xi=x_{1} y_{1} ; \quad \eta=\frac{x_{2}^{2}+y_{2}^{2}}{2}
$$


To get a symplectic change of coordinates in this theorem, except for results of [29] one needs to use Rüssmann's paper [34].

Remark 1. By a linear scaling of time and, if necessary, a canonical transformation $y_{1} \rightarrow$ $x_{1}, x_{1} \rightarrow-y_{1}$, one can obtain $\lambda=-1$, and $\omega>0$ (new $\omega$ is up to the sign the ratio $\left.|\omega / \lambda|\right)$. Later on we utilize this normalization.

Denote $\Phi^{t}: m \rightarrow \Phi^{t}(m)$ the flow generated by the vector field $X_{H}$. In the coordinates of Theorem 1 the system of differential equations is written down as

$$
\dot{x_{1}}=-h_{\xi} x_{1}, \quad \dot{y_{1}}=h_{\xi} y_{1}, \quad \dot{x_{2}}=-h_{\eta} y_{2}, \quad \dot{y_{2}}=h_{\eta} x_{2},
$$

and its flow $\Phi^{t}$ is

$$
\left(\begin{array}{l}
x_{1}(t) \\
y_{1}(t) \\
x_{2}(t) \\
y_{2}(t)
\end{array}\right)=\left(\begin{array}{cccc}
\exp \left[-t \cdot h_{\xi}^{0}\right] & 0 & 0 & 0 \\
0 & \exp \left[t \cdot h_{\xi}^{0}\right] & 0 & 0 \\
0 & 0 & \cos \left(t \cdot h_{\eta}^{0}\right) & -\sin \left(t \cdot h_{\eta}^{0}\right) \\
0 & 0 & \sin \left(t \cdot h_{\eta}^{0}\right) & \cos \left(t \cdot h_{\eta}^{0}\right)
\end{array}\right)\left(\begin{array}{l}
x_{1}^{0} \\
y_{1}^{0} \\
x_{2}^{0} \\
y_{2}^{0}
\end{array}\right)
$$

where notations are used

$$
h_{\xi}^{0}=h_{\xi}\left(\xi_{0}, \eta_{0}\right), \quad h_{\eta}^{0}=h_{\eta}\left(\xi_{0}, \eta_{0}\right), \quad \xi_{0}=x_{1}^{0} y_{1}^{0}, \quad \eta_{0}=\left(\left(x_{2}^{0}\right)^{2}+\left(y_{2}^{0}\right)^{2}\right) / 2 .
$$

The Hamiltonian system under consideration is reversible as well, hence it is important to understand to which simplest form can be reduced by means of the same symplectic coordinate change both the system and the involution in a neighborhood of a saddle-center. This was done in [8]. We remind the needed results.

Theorem 2. Let $X_{H}$ be an analytic Hamiltonian vector field and $p$ its equilibrium of the saddlecenter type. Suppose, in addition, $X_{H}$ be reversible w.r.t. the analytic anti-canonical involution $L$ and $p$ is symmetric. Then in some neighborhood $U$ of $p$ there are analytic coordinates, as in the Theorem 1, such that $L$ has one of two forms:

$$
\left(\begin{array}{l}
x_{1} \\
y_{1} \\
x_{2} \\
y_{2}
\end{array}\right) \rightarrow\left(\begin{array}{cccc}
0 & 1 & 0 & 0 \\
1 & 0 & 0 & 0 \\
0 & 0 & -1 & 0 \\
0 & 0 & 0 & 1
\end{array}\right)\left(\begin{array}{l}
x_{1} \\
y_{1} \\
x_{2} \\
y_{2}
\end{array}\right)
$$

or

$$
\left(\begin{array}{l}
x_{1} \\
y_{1} \\
x_{2} \\
y_{2}
\end{array}\right) \rightarrow\left(\begin{array}{cccc}
0 & -1 & 0 & 0 \\
-1 & 0 & 0 & 0 \\
0 & 0 & -1 & 0 \\
0 & 0 & 0 & 1
\end{array}\right)\left(\begin{array}{l}
x_{1} \\
y_{1} \\
x_{2} \\
y_{2}
\end{array}\right)
$$

\section{Local orbit structure near saddle-center}

Using Moser coordinates is the easiest way to describe the local topology of levels $V_{c}$ and the orbit behavior on each level [23]. The system locally near $p$ takes the form (1). There are two invariant symplectic disks: $x_{1}=y_{1}=0$ and $x_{2}=y_{2}=0$. Quadratic functions $\xi=x_{1} y_{1}$, $\eta=\left(x_{2}^{2}+y_{2}^{2}\right) / 2$ are local integrals of the system. Consider the momentum plane $(\xi, \eta)$ in a 
neighborhood of the origin $(0,0)$. The level $V_{c}$ of the Hamiltonian corresponds to the analytic curve $\xi=-c+\omega \eta+O\left(\eta^{2}+c^{2}\right)=a_{c}(\eta), 0 \leq \eta \leq \eta_{*}$. For $c$ small enough these curves form an analytic foliation of a neighborhood of the origin $(0,0)$. In fact, as $\eta \geq 0$, one needs to consider the rectangle $|\xi| \leq \xi_{0}, 0 \leq \eta \leq \eta_{*}$ in the momentum plane.

Consider first the level $V_{0}$, then we get the curve $\xi=\omega \eta+O\left(\eta^{2}\right)=a(\eta)$ on the momentum plane $(\xi, \eta), 0 \leq \eta \leq \eta_{*}$. To construct a neighborhood of the origin in $\mathbb{R}^{4}$ with coordinates $\left(x_{1}, y_{1}, x_{2}, y_{2}\right)$ we choose four cross-sections $\left|x_{1}\right|=d,\left|y_{1}\right|=d$. In the manifold $M$ a neighborhood $U$ of the point $p$ in coordinates $\left(x_{1}, y_{1}, x_{2}, y_{2}\right)$ can be thought as the direct product of two disks $\left(x_{1}, y_{1}, 0,0\right)$ and $\left(0,0, x_{2}, y_{2}\right)$.

The local structure of $V_{0}$ is investigated via its foliation into invariant levels of integral $\eta$. At $\eta=0$ (the origin on the disk $\left.\left(0,0, x_{2}, y_{2}\right)\right)$ we have $\xi=a(0)=0$, i.e. we get a "cross" on the disk $\left(x_{1}, y_{1}, 0,0\right)$ (the union of two segments $y_{1}=0$ and $\left.x_{1}=0\right)$ ). In $U$ the cross coincides with the union of local stable and unstable curves of the saddle-center $p$. For $\eta>0$ the value $\xi=a(\eta)$ is positive and on the disk $\left(x_{1}, y_{1}, 0,0\right)$ we get two pieces of the hyperbola $x_{1} y_{1}=a(\eta)$, which lie in the first and the third quadrants of the plane $\left(x_{1}, y_{1}, 0,0\right)$, respectively. In $U$ each piece of the hyperbola is multiplied on the circle $x_{2}^{2}+y_{2}^{2}=2 \eta$ on the plane $\left(0,0, x_{2}, y_{2}\right)$. Varying $\eta$ from zero till $\eta_{*}$, we get in $U$ two solid cylinders which have a unique common point, the origin, i.e. the saddle-center itself. Each solid cylinder contains the angle made up of two gluing semi-segments of the cross $\left(x_{1} \geq 0, y_{1}=0\right.$ and $y_{1} \geq 0, x_{1}=0$ for one cylinder and $x_{1} \leq 0$, $y_{1}=0$ and $y_{1} \leq 0, x_{1}=0$ for another cylinder). Each such angle is is the topological limit, as $\eta \rightarrow+0$, of cylinders $x_{1} y_{1}=a(\eta), x_{2}^{2}+y_{2}^{2}=2 \eta$, lying in the same solid cylinder. In particular, each of two solid cylinders contains one half of the stable curve (a stable separatrix) and one half of the unstable curve (unstable separtatrix) of the saddle-center. At the fixed $\eta>0$ each two-dimensional cylinder is an invariant set and orbits on it go from one of two cross-sections $\left|y_{1}\right|=d$ to another of two cross-sections $\left|x_{1}\right|=d$ (see. Fig. 2).

Remark 2. It is worth remarking the property that will be used below. In Moser coordinates on the level $H=0$ the cross-sections for orbits in the solid cylinder, which is projected onto the first quadrant of the plane $x_{2}=y_{2}=0$, are $y_{1}=d>0$ (for entering orbits) and $x_{1}=d$ (for outgoing orbits), but for the second solid cylinder, which is projected onto the third quadrant, they are $y_{1}=-d$ (for entering orbits) and $x_{1}=-d$ (for outgoing orbits mpaekmopuü). This implies that for the case of the first type involution symmetry permutes the orbit on $y_{1}>0, x_{1}=x_{2}=y_{2}=0$ with that on $x_{1}>0, y_{1}=x_{2}=y_{2}=0$ and orbit on $y_{1}<0, x_{1}=x_{2}=y_{2}=0$ with that on $x_{1}<0, y_{1}=x_{2}=y_{2}=0$. Hence the symmetry permutes cross-sections from the same solid cylinder. For the case the second type of involution the symmetry permuts the orbit on $y_{1}>0$, $x_{1}=x_{2}=y_{2}=0$ with that on $x_{1}<0, y_{1}=x_{2}=y_{2}=0$ and orbit on $y_{1}<0, x_{1}=x_{2}=y_{2}=0$ with that on $x_{1}>0, y_{1}=x_{2}=y_{2}=0$. Hence the symmetry permutes cross-sections from the different solid cylinders. This will be used below for the classification of heteroclinic connections.

A level $V_{c}$ as $c<0$ corresponds to the curve $\xi=-c+\omega \eta+O\left(\eta^{2}+c^{2}\right)=a_{c}(\eta)>0$ on the momentum plane $(\xi, \eta)$ for all $0 \leq \eta \leq \eta_{*}$. Therefore, the level $V_{c}$ as $c<0$ consists of two disconnected solid cylinders, their projections onto the momentum plane form two curvilinear rectangles in the first and third quadrants bounded by related segments $\left|x_{1}\right|=d,\left|y_{1}\right|=d$, and pieces of hyperbolas $x_{1} y_{1}=a_{c}(0)=-c>0$ and $x_{1} y_{1}=a_{c}\left(\eta_{*}\right)$. Each two-dimensional cylinder $\eta=\eta_{0}$ is foliated by flow orbits going from one cross-section $\left|y_{1}\right|=d$ to another one $\left|x_{1}\right|=d$ (see, Fig. 3).

For $c>0$ the situation is more complicated, since the curve $\xi=a_{c}(\eta)$ on the plane $(\xi, \eta)$ for 
$0 \leq \eta \leq \eta_{*}$ correspond to both positive and negative values of $\xi$ (we consider $|c|$ small enough). Denote $\eta_{c}$ the unique positive root of the equation $a_{c}(\eta)=0$. Then for $0 \leq \eta \leq \eta_{c}$ pieces of hyperbola $x_{1} y_{1}=a_{c}(\eta)<0$ belong to the second and fourth quadrants of the plane $\left(x_{1}, y_{1}, 0,0\right)$, but for $\eta>\eta_{c}$ they belong to the first and third quadrants of this plane. The topological type of the set $V_{c}$ is a connected sum of two solid cylinders (i.e. balls). To see this let us consider the projection of $V_{c}$ on the plane $\left(x_{1}, y_{1}, 0,0\right)$, it lies inside of the quadrate $\left|x_{1}\right| \leq d,\left|y_{1}\right| \leq d$. let us cut this set into two halves by the diagonal $y_{1}=-x_{1}$. Over this diagonal in $V_{c}$ a 2-dimensional sphere $S$ is situated. Indeed, extreme points of the diagonal in the second and fourth quadrants two points of $V_{c}$ correspond (for them $\eta=0$ ), but for any point of the diagonal between extreme points in $V_{c}$ a circle lies since $\eta>0$ for such points. In particular, over the point $(0,0)$ of the diagonal the circle $x_{2}^{2}+y_{2}^{2}=2 \eta_{c}$ lies, it is the Lyapunov periodic orbit $l_{c}$. Segments $x_{1}=0$ and, respectively, $y_{1}=0$ of the plane, in $V_{c}$ correspond to stable and unstable manifolds of the periodic orbits.

Subset $V_{c}^{+} \subset V_{c}$ lying over the half-plane $x_{1}+y_{1}>0$ is composed of three parts. One part corresponds to the values $\eta_{c} \leq \eta \leq \eta_{*}$. For the strict inequality we get a set being diffeomorphic to the direct product of an open annulus and a segment. As $\eta \rightarrow \eta_{c}+0$ this set has as a topological limit the set being the direct product of an angle in the plane $\left(x_{1}, y_{1}\right): 0 \leq x_{1} \leq d$, $y_{1}=0$ and $0 \leq y_{1} \leq d, x_{1}=0$, and a circle $x_{2}^{2}+y_{2}^{2}=2 \eta_{c}$. Two other parts of $V_{c}^{+}$are two solid cylinders. These are those subsets in $V_{c}^{+}$that are projected into the second and third quadrants of the plane $\left(x_{1}, y_{1}\right)$, they correspond to $\xi<0$. Every such a solid cylinder is foliated into two-dimensional cylinders lying over pieces of hyperbola $\xi=a_{c}(\eta)<0,0 \leq \eta<\eta_{c}, x_{1}+y_{1}>0$, respectively in the second and fourth quadrants. One of the bounding circles of this cylinder lies over the point on the diagonal (for each cylinder this point is own), the second bounding circle lies over the point on the segment $y_{1}=d$ or $x_{1}=d$. Solid cylinder projecting onto the second quadrant is glued with its lateral boundary to the set lying over the first quadrant along the cylinder $x_{1}=0, y_{1}>0, x_{2}^{2}+y_{2}^{2}=2 \eta_{c}$, but the second solid cylinder which is projecting onto the fourth quadrant, is glued by its lateral boundary to the set over the first quadrant along the cylinder $y_{1}=0, x_{1}>0, x_{2}^{2}+y_{2}^{2}=2 \eta_{c}$. Thus, the set is obtained that is homeomorphic to the solid cylinder from which an inner ball with the boundary $S$ is cut.

Similarly, the second part $V_{c}^{-}$of the set $V_{c}$ is obtained lying over half-plane $x_{1}+y_{1} \leq 0$. The sets $V_{c}^{-}$and $V_{c}^{+}$are glued along the sphere $S$, the gluing corresponds to the same points on the diagonal $y_{1}=-x_{1}$. Visually, this can be imagined in such a way that in each half (solid cylinder) we cut out by an inner ball and glue the halves obtained along the boundary of balls in accordance with their orientation. This is a particular case of a connected sum of two manifolds. Topologically the set obtained is homeomorphic to a spherical layer $S^{2} \times I$. Since each level is foliated into invariant cylinders $\eta=$ const, we get a complete picture of the local orbit behavior near a saddle-center (see Fig. 2,4).

\section{Poincaré map in a neighborhood of $\gamma$}

To describe the orbit behavior in a neighborhood of a periodic orbit $\gamma$ in the level $V_{0}$, we consider a two-dimensional symplectic analytic Poincaré map generated by the flow of $X_{H}$ on some analytic cross-section $\Sigma$ to $\gamma$. For the case under consideration, the vector field is reversible, hence the cross-section can be chosen in such a way that the reversibility would preserve for the Poincaré map, as well. 


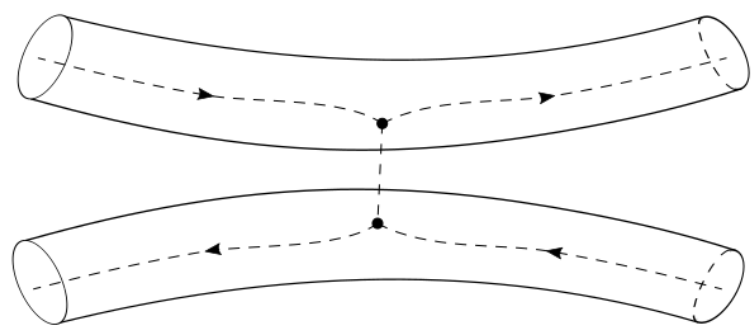

Figure 2: $c=0$

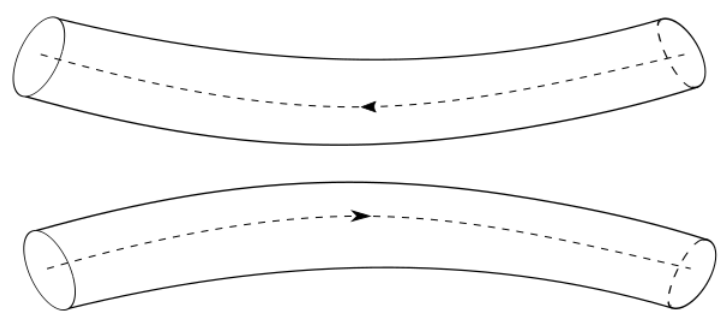

Figure 3: $c<0$

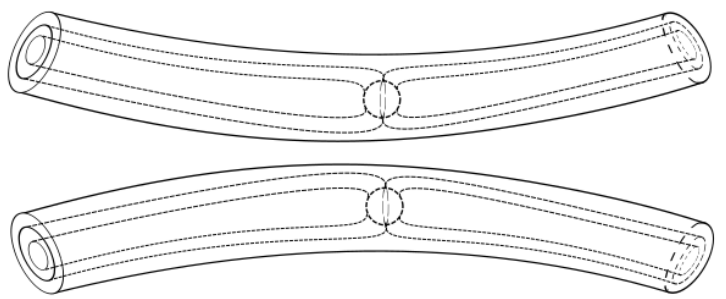

Figure 4: $c>0$

A symmetric periodic orbit intersects submanifold $F i x(L)$ at two points $m_{1}, m_{2}$. Take one of them, $m=m_{1}$, and consider a three-dimensional analytic cross-section $N$ for $\gamma$ containing $m$. $N$ can be chosen in such a way that $m$ belongs to $N$ along with some sufficiently small analytic disk from $\operatorname{Fix}(L)$ and $N$ is invariant w.r.t. the action of $L$. We assume further such choice of the cross-section.

In a sufficiently small neighborhood of $m$ levels $V_{c}$ form an analytic foliation into threedimensional submanifolds since $d H_{m} \neq 0$. The level $V_{0}$ contains the curve $\gamma$, but $N$ is transversal to the curve, hence $V_{0}$ and $N$ intersect each other transversely at the point $m$ and therefore they intersect in $M$ along an analytic 2-dimensional disk $\Sigma \subset N$. $\Sigma$ is a cross-section to $\gamma$ in the level $V_{0}$ and we get an analytic Poincaré map $S: \Sigma \rightarrow \Sigma$ with a saddle fixed point $m$.

To study orbit behavior of a system in a neighborhood of $\gamma$ we use Moser theorem [30] on the normal form of a 2-dimensional analytic symplectic diffeomorphism near its saddle fixed point. As $\gamma$ is orientable by the assumption, its multipliers $\nu, \nu^{-1}$ are positive. 
Theorem 3 (Moser). In a neighborhood of a saddle fixed point of a real analytic symplectic diffeomorphism $S$ there are analytic symplectic coordinates $(u, v)$ and an analytic function $f(\zeta)$, $\zeta=u v, f(0)=\nu$, such that $S$ takes the following form

$$
\bar{u}=u / f(\zeta), \bar{v}=v f(\zeta), \text { where } f(\zeta)=\nu+O(\zeta), 0<\nu<1 .
$$

For our case $S$ is also reversible w.r.t. the restriction of the involution on $\Sigma$, and involution permutes stable and unstable manifolds (here - curves) of the fixed point $m$. As $X_{H}(m) \neq 0$, then intersection of Fix $(L)$ and $V_{0}$ is transverse at $m$ and it is an analytic curve $l \subset \Sigma$ being the symmetry line containing $m$. It is not hard to prove, following [10] that symplectic coordinates $(u, v)$ in the Moser theorem can be chosen in such a way that the restriction of the involution on $\Sigma$ would act as $(u, v) \rightarrow(v, u)$. Then the fixed point set of the involution near point $m$ coincides with the diagonal $u=v$. We assume henceforth this to hold.

The orbit $\Gamma_{1}$ is nonsymmetric and approaches $\gamma$ as $t \rightarrow-\infty$, hence it intersects $\Sigma$ at a countable set of points tending to $m$, but not lying on $l$. These points belong to the analytic curve $w_{u}$ being the trace on the disk $\Sigma$ of manifold $W^{u}(\gamma)$. Similarly, the orbit $\Gamma_{2}$ intersects $\Sigma$ at countable set of points approaching $m$ at positive iterations of $S$, the points do not lie on $l$. These points belong to the analytic curve $w_{s}$ being the trace of the manifold $W^{s}(\gamma)$ on $\Sigma$. In Moser coordinates local stable curve coincides with the axis $v$ (it is given as $u=0$ ), and local unstable one with the axis $u(v=0)$. Therefore the point $p_{s}$, the trace of $\Gamma_{2}$, has coordinates $\left(0, v_{+}\right)$, and $p_{u}=L\left(p_{s}\right)$ is the trace of $\Gamma_{1}$ and has coordinates $\left(u_{-}, 0\right)$. To be definite, we assume $v_{+}>0$. Then, due to reversibility, one has $u_{-}=v_{+}$.

We choose neighborhoods $\Pi^{s}, \Pi^{u}$ of point $p_{s}, p_{u}$ defined by inequalities $\Pi^{s}:\left|v-v_{+}\right|<\varepsilon,|u|<$ $\delta$, и $\Pi^{u}:|v|<\delta,\left|u-u_{-}\right|<\varepsilon$, the quantities $\delta, \varepsilon$ are small enough. The set of points from $\Pi^{s}$ which are transformed to $\Pi^{u}$ by some iteration of the map $S$, as is known [40, 35], consists of the countable set of strips in $\Pi^{s}$ accumulating to the stable curve $u=0$. Due to a convenient normal form, these strips are easily found. The following assertion holds

Lemma 1. Equations $u=f^{k}(\zeta)\left(u_{-} \pm \varepsilon\right)$ define functions $u=s_{k}^{ \pm}(v)$, whose domain is $\left|v-v_{+}\right|<$ $\varepsilon$. For them inequalities $s_{k}^{+}(v)>s_{k}^{-}(v)$ hold true $s_{k}^{-}(v)>s_{k+1}^{+}(v)$, and $s_{k}^{+}(v)$ are uniformly tend to zero as $k \rightarrow \infty$.

Proof. The proof of this lemma is evident. The lateral boundaries of strips $\sigma_{k}^{s}$ are segments $\left|v-v_{+}\right|= \pm \varepsilon$. Its upper boundary serves the curve $s_{k}^{+}(v)$ providing by solution of the equation $u_{k}=u_{-}+\varepsilon$, and lower boundary is the curve being the solution of the equation $u_{k}=u_{-}-\varepsilon$. To prove the lemma we take an arbitrary $v,\left|v-v_{+}\right| \leq \varepsilon$ and find the related values $u=s_{k}^{+}(v)$ and $u=s_{k}^{-}(v)$ from the equations:

$$
u=f^{k}(\zeta)\left(u_{-}+\varepsilon\right), u=f^{k}(\zeta)\left(u_{-}-\varepsilon\right) .
$$

Consider, for example, the first equation. Since the value of $\zeta$ preserves along the orbit of $S$, then multiplying both sides of the first equation at $v$, we get $g_{k}(\zeta)=\zeta / f^{k}(\zeta)=v\left(u_{-}+\varepsilon\right)$. For $k \geq k_{0}>0$ this sequence of functions complex functions has each the inverse one and all of them are defined in the same disk $|\zeta| \leq \sigma$ of the complex plane $\mathbb{C}$. This follows from the complex inverse function theorem, sinc $g_{k}(0)=0, g_{k}^{\prime}(0)=\nu^{-k}$. Thus we get $s_{k}^{+}(v)=g^{-1}\left(v\left(u_{-}+\varepsilon\right)\right) / v$ and $s_{k}^{+}(v) \rightarrow 0$ as $k \rightarrow \infty$ uniformly in $v$. 
Functions $s_{k}^{ \pm}(v)$ are upper and lower boundaries of the strip $\sigma_{k}^{s}$. It implies the existence of a countable set of such strips

$$
k>k_{0}=E\left\{\frac{\ln \left(\left(\varepsilon+u_{-}\right) / \delta\right)}{\ln \left(\nu^{-1}\right)}\right\} .
$$

Here it is assumed $\varepsilon+u_{-}>\delta$ (this is the first restriction on the quantities $\varepsilon, \delta$ ).

The restriction of $L$ on $\Sigma$ acts as $L:(u, v) \rightarrow(v, u)$, hence we get $u_{-}=v_{+}=r$. Thus we have the same condition on $k_{0}$ for strips $\sigma_{k}^{u}$ :

$$
k>k_{0}=E\left\{\frac{\ln ((\varepsilon+r) / \delta}{\ln \left(\nu^{-1}\right)}\right\} .
$$

One more restriction on these quantities provides the requirement that the neighborhood $\Pi^{s}$ would not intersect with its image $S\left(\Pi^{s}\right)$, and $\Pi^{u}$ with its pre-image $S^{-1}\left(\Pi^{u}\right)$, and $\Pi^{s} \cap \Pi^{u}=\emptyset$. These conditions lead to the inequalities:

$$
\delta<r \frac{1-\nu}{1+\nu}, \quad \delta<r-\varepsilon .
$$

Now we can assert, due to construction, that all orbits of the map $S$, which pass through the points of the neighborhood $\Pi^{s}$ and reach the neighborhood $\Pi^{u}$ for positive iterations, have to pass through one of strips $\sigma_{k}^{s}, k \geq k_{0}$. these strips has, as its topological limit, the segment $u=0$ in $\Pi^{s}$. Its points belong to the stable manifold and they tend under $S^{n}$ to the fixed point $m$ as $n \rightarrow \infty$.

From the reversibility of $S$ and the same considerations we get that images of strips $\sigma_{k}^{s}$ in $\Pi^{u}$, i.e. strips $\sigma_{k}^{u}$, accumulate as $k \rightarrow \infty$ to the points of the segment $v=0$ in $\Pi^{u}$. These points under negative iterations of $S$ tend to $m$.

Remark 3. It is worth remarking the useful fact. At a given small value of $c$ the Hamiltonian system in a neighborhood of $\gamma$ has an analytic invariant 3-dimensional submanifold $V_{c}=\{H=c\}$. In $V_{c}$ a saddle periodic orbit $\gamma_{c}$ lies being a continuation in $c$ of the orbit $\gamma$. The family $\gamma_{c}$ makes up an analytic 2-dimensional local symplectic cylinder containing $\gamma_{0}=\gamma$. Chosen above 3-dimensional cross-section $N$ under its intersection with $V_{c}$ gives an analytic 2-dimensional symplectic disk $\Sigma_{c}$ being the local cross-section for the restriction of the flow on $V_{c}$. The Poincaré map $S_{c}$ on $\Sigma_{c}$ is symplectic analytic with the saddle fixed point $m_{c}$. For this map Moser theorem as also valid and its can be transformed to the form (3). Moreover, since the dependence on $c$ is analytic the change of variables can be done for all $c$ small enough at once and in (3) function $f$ will depend on $c$ analytically. This will be used below to study the dynamics on $V_{c}$ for $c$ close to $c=0$.

\section{Global maps}

Now we derive the representations of the global maps $T_{1}$ generated by the flow near $\Gamma_{1}$ acting as $T_{1}: \Pi^{u} \rightarrow D_{1}$. It is analytic symplectic diffeomorphism and is written as $x_{2}=f(u, v), \quad y_{2}=$ $g(u, v)$, here symplecticity is equivalent to the identity $f_{u} g_{v}-f_{v} g_{u} \equiv 1$ (area preservation).

Linearization of this map at the point $\left(u_{-}, 0\right)$ has the form $x_{2}=\alpha\left(u-u_{-}\right)+\beta v, y_{2}=$ $\gamma\left(u-u_{-}\right)+\delta v$, where $\alpha=f_{u}, \quad \beta=f_{v}, \quad \gamma=g_{u}, \quad \delta=g_{v}$, all derivatives are calculated at the point $\left(u_{-}, 0\right)$. Thus, a general form of $T_{1}$ is

$$
x_{2}=\alpha\left(u-u_{-}\right)+\beta v+\cdots, \quad y_{2}=\gamma\left(u-u_{-}\right)+\delta v+\cdots .
$$


The system under study is reversible and cross-sections are chosen consistently with the action of involution, then the global map $T_{2}: D_{2} \rightarrow \Pi^{s}$ near $\Gamma_{2}=L\left(\Gamma_{1}\right)$ is expressed as $T_{2}=L \circ T_{1}^{-1} \circ L$, or in coordinates:

$$
\begin{array}{r}
u_{1}=\gamma \bar{x}_{2}+\alpha \bar{y}_{2}+\cdots, \\
v_{1}-v_{+}=-\delta \bar{x}_{2}-\beta \bar{y}_{2}+\cdots .
\end{array}
$$

Below, when studying of the orbit behavior on the levels $V_{c}$ for $c \neq 0$, we shall need to know the form of the global maps in these cases. As was mentioned above, without loss of generality, we can regard as coordinates on the disks $D_{1}(c), D_{2}(c)$ symplectic coordinates $\left(x_{2}, y_{2}\right)$ and $\left(\bar{x}_{2}, \bar{y}_{2}\right)$, respectively, and on the disk $\Sigma(c)$ symplectic coordinates $(u, v)$. Global maps are analytic symplectic diffeomorphisms analytically depending on $c$. Thus they have the form

$$
\begin{aligned}
& T_{1}(c): x_{2}=a(c)+\alpha(c)\left(u-u_{-}\right)+\beta(c) v+\cdots, y_{2}=b(c)+\gamma(c)\left(u-u_{-}\right)+\delta(c) v+\cdots \\
& T_{2}(c): u_{1}=a_{1}(c)+\gamma(c) \bar{x}_{2}+\alpha(c) \bar{y}_{2}+\cdots, v_{1}-v_{+}=b_{1}(c)-\delta(c) \bar{x}_{2}-\beta(c) \bar{y}_{2}+\cdots
\end{aligned}
$$

where $a_{1}(c)=\gamma(c) a(c)-\alpha(c) b(c), b_{1}(c)=-\delta(c) a(c)+\beta(c) b(c)$.

\section{Types of symmetric connections}

The symmetric periodic orbit $\gamma$ in the level $V_{0}$ is outside of a neighborhood of point $p$, so one needs to conform the location of this orbit and its symmetry with the action of involution in $U$ relative to coordinates. This concordance is relied on the existence of connecting orbits $\Gamma_{1}$ and $\Gamma_{2}=L\left(\Gamma_{1}\right)$.

Near the point $p$ involution $L$ permutes local stable and unstable curves of $p$. Since heteroclinic orbits contain these curves, two cases are possible here. To understand this we remind that we have chosen in $U$ on the level $V_{0}$ two smooth disks $D_{1}, D_{2}=L\left(D_{1}\right)$ being transverse to orbits $\Gamma_{1}$ and $\Gamma_{2}$, respectively. In Moser coordinates near $p$ we can take as such disks cross-sections $y_{1}= \pm d$ and $x_{1}= \pm d$, where the sign is determined by the intersection of a respective crosssection with $\Gamma_{1}$ and $\Gamma_{2}$. On $V_{0}$ coordinates on the disks are $\left(x_{2}, y_{2}\right)$, since a coordinate conjugated to $y_{1}$ (or, respectively, to $x_{1}$ ) is found from the equality $H=0$. Recall (see above) that in a neighborhood of point $p$ the local topological type of the level $V_{0}$ is a pair of 3-dimensional solid cylinders with two their inner points identified chosen by one in each cylinder (after gluing this is the point $p$ ) (see Fig. 2). The lateral boundary of each solid cylinder is a smooth 2-dimensional invariant cylinder, two other boundaries are two disks ("lids"). For each solid cylinder orbits enter through one lid and leave the cylinder through the other lid.

One can regard the cross-sections $D_{1}, D_{2}$ be two lids of these cylinders and what is more, $D_{1}$ is the entry disk and $D_{2}$ is exit one. Two different situations are possible: 1) both orbits $\Gamma_{1}, \Gamma_{2}$ belong locally to the same solid cylinder, this is equivalent to the conditions that both disks $D_{1}, D_{2}$ are on the boundary of the same cylinder; 2) orbit $\Gamma_{1}$ belongs locally to one solid cylinder but $\Gamma_{2}$ belongs locally to another solid cylinder, that is, disks $D_{1}$ and $D_{2}$ belong to the boundaries of different cylinders. In Moser coordinates the case 1 corresponds to the action $L:\left(x_{1}, y_{1}, x_{2}, y_{2}\right) \rightarrow\left(y_{1}, x_{1},-x_{2}, y_{2}\right)$, but the case 2 does to the action $L:\left(x_{1}, y_{1}, x_{2}, y_{2}\right) \rightarrow$ $\left(-y_{1},-x_{1},-x_{2}, y_{2}\right)$. The case 1 means the invariance of the related cylinder w.r.t. the involution and the case 2 means its permutability (one cylinder transforms to another one). In case if $L$ 
preserves the cylinder, the intersection of $\operatorname{Fix}(L)$ with the cylinder is a curve, but if $L$ permutes cylinders this intersection is the only point $p$ (see, Fig. 5, 6).

Now consider those orbits of the vector field which enter through $D_{1}$ near $\Gamma_{1}$, but differ from $\Gamma_{1}$. As $t$ increases, they enter into the related solid cylinder, pass it and leave the cylinder (the semi-orbit $\Gamma_{1}$ itself tends to $p$ and stays in the cylinder). These orbits either intersect $D_{2}$ (case 1 ), or leave the cylinder without intersecting $D_{2}$ (case 2). In the case 2 the Poincaré map is not defined in a neighborhood of the connection for $c \leq 0$, since orbits close to $\Gamma_{1}$ do not return on $D_{2}$, if a system under consideration does not fulfil some additional global conditions (of the type the existence of a homoclinic orbit joining two remaining lids).

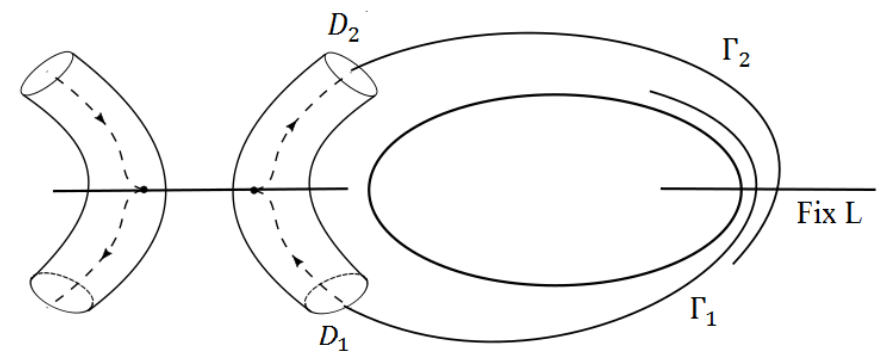

Figure 5: Case 1

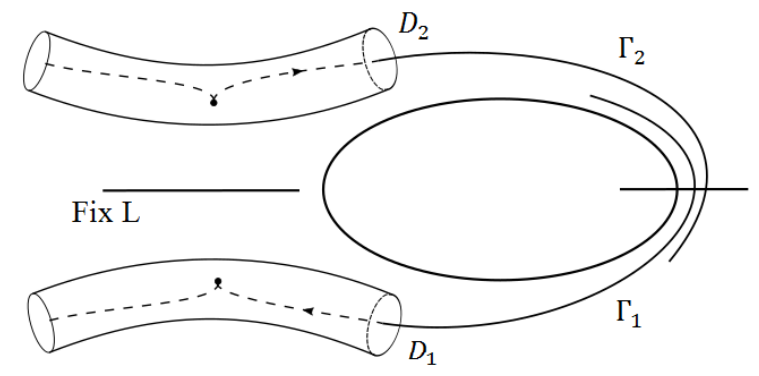

Figure 6: Case 2

Remark 4. For $c>0$ small enough for the case 2 the Poincaré map on the related disk $D_{1}(c)$ becomes defined inside of some small disk centered at $(0,0)$ whose boundary circle is the trace of a stable manifold $W^{s}\left(l_{c}\right)$ of the Lyapunov periodic orbit $l_{c}$. Then for some sequence of positive $c_{n} \rightarrow 0$ the trace on the disk $D_{1}\left(c_{n}\right)$ of $W^{s}\left(\gamma_{c_{n}}\right)$ is tangent to the trace of $W^{u}\left(\gamma_{c_{n}}\right)$, what is accompanied with the appearance of elliptic periodic orbits. This will be considered below.

\section{In the singular level $V_{0}$}

We start with the case 1 at $c=0$. In the neighborhood $U$ of $p$ we have in Moser coordinates the representation $h=-\xi+\omega \eta+R(\xi, \eta)$, hence the manifold $W^{c s}$ is given as $x_{1}=0, W^{c u}$ as $y_{1}=0$, $W^{s}$ by the equalities $x_{1}=x_{2}=y_{2}=0$, and $W^{u}$ by $y_{1}=x_{2}=y_{2}=0$. Suppose, to be definite, 
that in $U$ heteroclinic orbit $\Gamma_{1}$ approaches to $p$ for values $y_{1}>0$, i.e. in the level $V_{0}$ disk $D_{1}$ is defined by the equality $y_{1}=d$ and disk $D_{2}$ by the equality $x_{1}=d$. In $U$ signs of variables $x_{1}$ and $y_{1}$ preserve by the flow and 3-dimensional cross-section $N^{s}: y_{1}=d>0,\left|x_{1}\right| \leq \delta, \eta \leq \eta_{0}$, is transverse to $\Gamma_{1}$ and to all orbits close to $\Gamma_{1}$, due to the inequality $h_{\xi}=-1+\ldots \neq 0$ in $U$. Similar assertions are valid for the cross-section $N^{u}=L\left(N^{s}\right): x_{1}=d,\left|y_{1}\right| \leq \delta, \eta \leq \eta_{0}$. Each cross-section is foliated by levels $H=c$ into disks, one of which is $D_{1}=V_{0} \cap N^{s}$ and, respectively, $D_{2}=V_{0} \cap N^{u}$.

Denote as $a(\eta)$ the solution of the equation $h(\xi, \eta)=0$ w.r.t. $\xi, \xi=a(\eta)=\omega \eta+O\left(\eta^{2}\right)$. One may regard that when variables $(x, y)$ vary in $U \cap V_{0}$, corresponding solutions of the equation $h(\xi, \eta)=0$ lie on the graph of function $a$. Then 2-disk $D_{1}$ in $N^{s}$ is the graph of the function $x_{1}=a(\eta) / d$, and 2-disk $D_{2}$ in $N^{u}$ is the graph of function $y_{1}=a(\eta) / d$. Both $D_{1}, D_{2}$ are analytic disks being symplectic w.r.t. the restriction of 2 -form $\Omega$ on $D_{1}$ and $D_{2}$, respectively, and the local map $T: D_{1} \rightarrow D_{2}$ generated by the flow $\Phi^{t}$ is symplectic.

Remark 5. For the type 2 of the involution the cross-sections are $y_{1}=d$ (for $N^{s}$ ) and $x_{2}=-d$ $\left(\right.$ for $\left.N^{u}\right)$. Therefore, orbits from $D_{1}=N^{s} \cap V_{0}$ hit $D_{2}=N^{u} \cap V_{0}$ only if $a(\eta)<0$.

Let us find an explicit representation of the map $T$ in coordinates $\left(x_{2}, y_{2}\right)$. The passage time $\tau$ for orbits from $N^{s}$ to $N^{u}$ is found from (1), where $x_{1}(\tau)=d, y_{1}(0)=d$ : $\tau=$ $-\left(h_{\xi}\right)^{-1} \ln \left(d / x_{1}\right), x_{1}=a(\eta) / d$. From (1) it follows that $T$ has the form

$$
\bar{x}_{2}=x_{2} \cos \Delta(\eta)-y_{2} \sin \Delta(\eta), \quad \bar{y}_{2}=x_{2} \sin \Delta(\eta)+y_{2} \cos \Delta(\eta),
$$

with

$$
\Delta(\eta)=-\frac{h_{\eta}}{h_{\xi}} \ln \left(d / x_{1}\right)=a^{\prime}(\eta) \ln \left(d^{2} / a(\eta)\right)=(\omega+O(\eta)) \ln \left(d^{2} / a(\eta)\right) .
$$

Remark 6. For the type 2 of the involution the formula is modified as $\Delta(\eta)=a^{\prime}(\eta) \ln \left(-d^{2} / a(\eta)\right)$.

Our first result is the following theorem

Theorem 4. If an analytic reversible Hamiltonian system has a heteroclinic connection of the type 1 with properties indicated, then the saddle periodic orbit $\gamma$ has a countable set of 1-round transverse homoclinic orbits. In the case of the type 2 connection no other orbits exist in $V_{0}$ in a sufficiently small neighborhood of the connection, except for orbits of the connection.

To be precise, let us make more exact the notion of a 1-round homoclinic orbit for $\gamma$. To this end, consider in $V_{0}$ a sufficiently small tubular neighborhood of the orbit $\gamma$. Since $V_{0}$ is orientable, this neighborhood is homeomorphic to a solid torus $D^{2} \times S^{1}$. The union of point of the orbit $\Gamma_{1}$, point $p$ and points of the orbit $\Gamma_{2}$ give a simple non-closed curve without selfintersections in $V_{0}$. One may regard that this infinite curve consists of three connected pieces, one of which, $R$, lies outside of the tubular neighborhood of $\gamma$, and two remaining ones are inside of this tubular neighborhood (recall that orbits $\Gamma_{1}, \Gamma_{2}$ tend asymptotically to $\gamma$ ). Now consider a homoclinic orbit to $\gamma$, whose global part outside of the tubular neighborhood of $\gamma$ belongs to a small neighborhood of the curve $R$, but two remaining parts are inside of the tubular neighborhood. Such homoclinic orbit for $\gamma$ will be called 1-round one.

Proof. To prove the theorem we will show that a segment of the unstable separatrix $w_{u} \cap \Pi^{u}$ of the saddle fixed point $m$ on $\Sigma$ is transformed by the the map $T_{2} \circ T \circ T_{1}$ into an analytic curve 
that intersects transversely at the countable set of points the segment $w_{s} \cap \Pi^{s}$ of the unstable separatrix $w_{s}$ of the same fixed point.

Consider in $\Pi^{u}: v=0,\left|u-u_{-}\right| \leq \varepsilon_{1}<\varepsilon$, a segment $(A, B)$ of the curve $w_{u}$. Its image under the action of $T_{1}$ is a parameterized curve on the disk $D_{1}: x_{2}(\tau)=\alpha \tau+\cdots, y_{2}(\tau)=\gamma \tau+\cdots$, its parameter is $\tau=u-u_{-}$. Since $T_{1}$ is a diffeomorphism, we get a smooth curve in $D_{1}$ passing through $(0,0)$, its tangent vector at $(0,0)$ is nonzero vector $(\alpha, \gamma)$. Boundary points of this curve denote as $A_{1}, B_{1}$ and the curve obtained as $\left[A_{1}, B_{1}\right]$.

The curve $\left[A_{1}, B_{1}\right]$ by the map $T$ is transformed to the spiral-shape curve on the disk $D_{2}$ :

$$
\begin{aligned}
& \bar{x}_{2}=x_{2}(\tau) \cos \Delta(\eta(\tau))-y_{2}(\tau) \sin \Delta(\eta(\tau)), \\
& \bar{y}_{2}=x_{2}(\tau) \sin \Delta(\eta(\tau))+y_{2}(\tau) \cos \Delta(\eta(\tau)) .
\end{aligned}
$$

In symplectic polar coordinates on $D_{1}, D_{2}$, respectively,

$$
x_{2}=\sqrt{2 \eta} \cos \phi, \quad y_{2}=\sqrt{2 \eta} \sin \phi, \bar{x}_{2}=\sqrt{2 \bar{\eta}} \cos \theta, \quad \bar{y}_{2}=\sqrt{2 \bar{\eta}} \sin \theta,
$$

the map $T$ has the form

$$
\bar{\eta}=\eta, \quad \theta=\phi+\Delta(\eta)(\bmod 2 \pi) .
$$

This map is defined for values $\eta>0$. Under the action of $T$ the curve $\left[A_{1}, B_{1}\right]$ transforms into two infinite spirals corresponding to $\tau>0$ and $\tau<0$

$$
\bar{\eta}=\eta(\tau), \quad \theta=\phi(\tau)+\Delta(\eta(\tau)),
$$

where for $|\tau|$ small enough we have for $\alpha \neq 0$

$$
\eta(\tau)=\left(x_{2}^{2}(\tau)+y_{2}^{2}(\tau)\right) / 2=\frac{\alpha^{2}+\gamma^{2}}{2} \tau^{2}+O\left(\tau^{3}\right), \tan \phi(\tau)=\frac{\gamma}{\alpha}+O(\tau),
$$

and for $\alpha=0$ the angle is defined via $\cot \phi$, here the values $\phi$ as $\tau \rightarrow+0$ and $\tau \rightarrow-0$ differ by $\pi$. Since $\phi$ is bounded as $\tau \rightarrow \pm 0$, but function $\Delta(\eta(\tau))$ monotonically increase to $\infty$, then each of spirals, as $|\tau| \rightarrow 0$, tends to $(0,0)$ on $D_{2}$, making infinite number of rotations in angle: $\theta(\tau) \rightarrow \infty$. Take a segment on $u=0$ symmetric to $[A, B]$ and its $T_{2}$-pre-image $\left[A_{2}, B_{2}\right]$ on $D_{2}$, it is an analytic segment through the point $(0,0)$ symmetric to $\left[A_{1}, B_{1}\right]$. Therefore, it intersects each spiral at the countable set of points through which orbits pass tending to $\gamma$ as $t \rightarrow \pm \infty$, that is, they are Poincaré homoclinic orbits [35]. To complete the proof, we need to show transversality of intersections spirals and $\left[A_{2}, B_{2}\right]$. Instead, we shall prove the transversality of $T_{2}$-images of spirals and the segment $u=0$ on $\Pi^{s}$.

Consider, for instance, one of spirals, defined by inequality $\tau>0$ and apply $T_{2}$

$$
\begin{array}{r}
u_{1}=\gamma \bar{x}_{2}+\alpha \bar{y}_{2}+\cdots=\sqrt{2 \eta(\tau)} \sqrt{\alpha^{2}+\gamma^{2}}[\sin (\varphi(\tau)+\Delta(\eta(\tau))+\sigma)+O(\sqrt{2 \eta(\tau)})], \\
v_{1}-v_{+}=-\delta \bar{x}_{2}-\beta \bar{y}_{2}+\cdots=\sqrt{2 \eta(\tau)} \sqrt{\beta^{2}+\delta^{2}}\left[\sin \left(\varphi(\tau)+\Delta(\eta(\tau))+\sigma_{1}+O(\sqrt{2 \eta(\tau)})\right] .\right.
\end{array}
$$

The map $T_{2}$ transforms the spiral and the point $(0,0)$ from $D_{2}$ to some spiral-shape curve and the point $\left(0, v_{+}\right)$in $\Pi^{s}$. We need to prove that the spiral obtained does not tangent to the segment $u=0$ at any common point. To this end, we show that the derivative $u_{1}^{\prime}(\tau)$ does not vanish at the intersection points of the spiral with the segment $u=0$ in $\Pi^{s}$. As $\eta(\tau) \neq 0$, zeros of the function $u_{1}(\tau)$ are determined by zeros of the function $\sin (\varphi(\tau)+\Delta(\eta(\tau))+\sigma)$, and one needs to check the inequality $u_{1}^{\prime}(\tau) \neq 0$ for those $\tau$ where $u_{1}=0$. 
The derivative $u_{1}^{\prime}(\tau)$ at points where $\sin (\varphi(\tau)+\Delta(\eta(\tau))+\sigma)=0$, is equal up to a nonzero multiplier

$$
\cos (\varphi(\tau)+\Delta(\eta(\tau))+\sigma)\left(\varphi^{\prime}(\tau)+\Delta^{\prime}(\eta(\tau)) \eta^{\prime}(\tau)\right) .
$$

Thus, the first multiplier is nonzero and the principal term in the bracket for small enough $\tau$ is $\Delta^{\prime}(\eta(\tau)) \eta^{\prime}(\tau)$, that tends to infinity as $\tau \rightarrow 0$. Indeed, in accordance to formula 6 for $\Delta$ we have

$$
\Delta^{\prime}(\eta)=a^{\prime \prime}(\eta) \ln \left(d^{2} / a(\eta)\right)-\frac{a^{\prime 2}(\eta)}{a(\eta)}=\frac{-a^{\prime 2}(\eta)+a^{\prime \prime}(\eta) a(\eta) \ln \left(d^{2} / a(\eta)\right)}{a(\eta)},
$$

hence the numerator is negative and separated from zero for small $\eta$, but the denominator tends to zero as $\eta \rightarrow+0$. The ratio $\eta^{\prime}(\tau) / a(\eta(\tau))$ is of the order $1 / \tau$. Therefore, the existence of a countable set of transverse homoclinic orbits has been proved.

For the case 2 orbits of the system passing on $\Pi^{u}$ through the points of unstable curve $v=0$, $\left|u-u_{-}\right|<\varepsilon$, as $t$ increases, intersect disk $D_{1}$ and after that leave $V_{0}$ (see Fig. 6). The same holds true, due to symmetry, as $t$ decreases, for orbits passing on $\Pi^{s}$ through the points of stable curve $u=0,\left|v-v_{+}\right|<\varepsilon$.

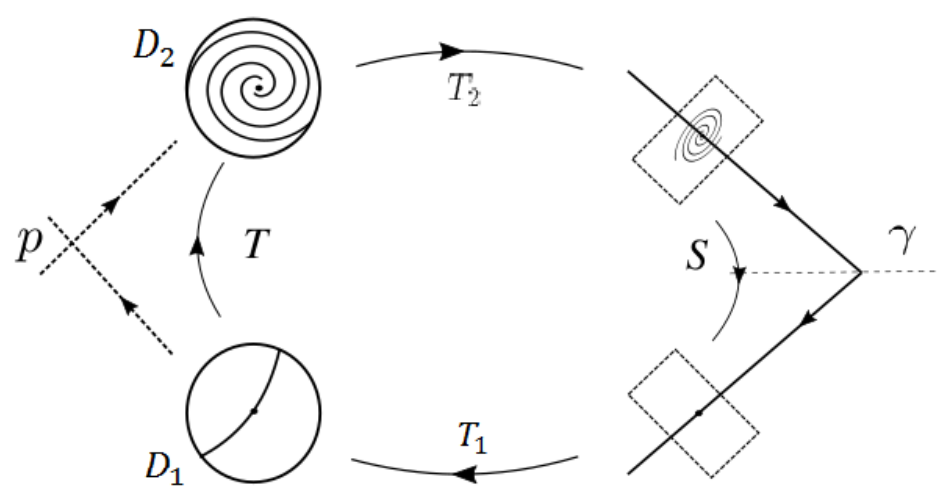

Figure 7: Poincaré map as $c=0$.

The proven theorem allows one to use results [40, 35] about the orbit structure near a transverse homoclinic orbit of a two-dimensional diffeomorphism. Namely, near each homoclinic orbit there exists its neighborhood such that orbits of a diffeomorphism passing through this neighborhood make up an invariant hyperbolic subset whose dynamics is conjugated with the shift on a transitive Markov chain (see, for instance, [7]). On $\Pi^{s}$ we have a countable set of different homoclinic orbits accumulating at the trace of heteroclinic orbit $\Gamma_{2}$. It is clear, for a fixed a homoclinic point from the set the size of a neighborhood, where the description holds, tends to zero as homoclinic points approach to the trace of $\Gamma_{2}$. If we consider an only finite number of homoclinic points outside of a small neighborhood of the trace of $\Gamma_{2}$, then we get a uniformly hyperbolic set generated by these homoclinic orbits. Therefore, the entire region where hyperbolic set for our case exists should be of a two-horn shape bounded by two parabola-like curves which are tangent at the point $\left(0, v_{+}\right)$. The strips near homoclinic orbits (see above) for different homoclinic points interact each other under iterations of the Poincaré map that lead to a Markov chain with a countable set of states, but the invariant set obtained in this way is not uniformly hyperbolic but only non-uniformly hyperbolic. 


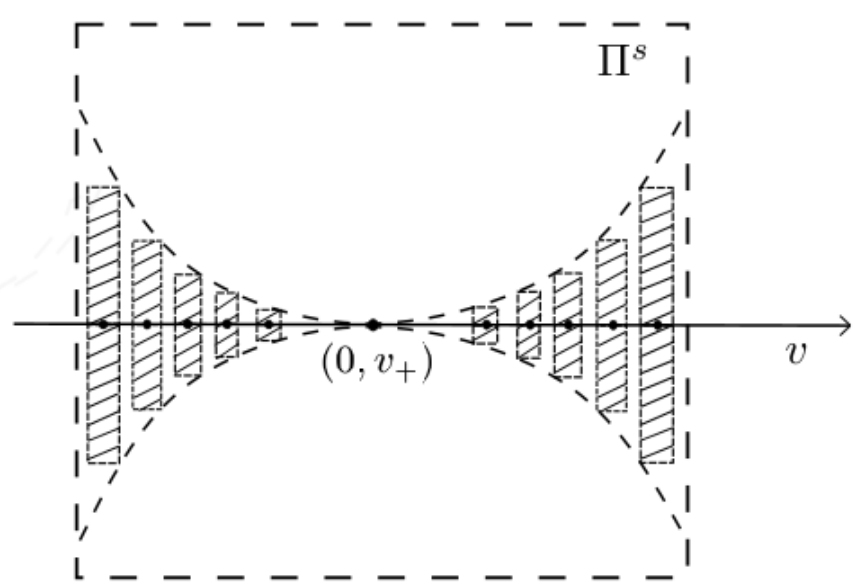

Figure 8: The shape of non-uniform hyperbolicity region in $\Pi^{s}$.

\section{$9 \quad$ Hyperbolicity and ellipticity in levels $c<0$}

In this section we consider levels $V_{c}, c<0$, near the connection for the case 1 . For the case 2 and $c<0$ all orbits, entering through $D_{1}(c)$ to a neighborhood, leave it, the same is true for the orbits entering a neighborhood, as $t$ decreasing, through $D_{2}(c)$.

We prove: 1) existence of a hyperbolic set constructed on a finite number of transverse homoclinic orbits to the saddle periodic orbit $\gamma_{c} ; 2$ ) existence of a countable set of intervals of values $c<0$ accumulating at zero whose values of $c$ correspond to levels where in $V_{c}$ a 1-round elliptic periodic orbit exists. Remind that for the case 1 and negative $c$ small enough all orbits in a neighborhood of $p$ passing through one "lid" $D_{1}(c)=N^{s} \cap V_{c}$ of the solid cylinder, as $t$ increases, intersect its second lid $D_{2}(c)=N^{u} \cap V_{c}$.

Existence of finitely many transverse homoclinic orbits to a periodic orbit $\gamma_{c}$ is almost evident and follows from their existence at $c=0$. For any negative $c$ small enough we consider that solid cylinder of the local part of the level $V_{c}$ near $p$, whose lids are $D_{1}(c), D_{2}(c)$. Then global maps transform: a segment $w_{u}(c)$ on $\Pi^{u}(c)$ (i.e. $v=0$ ) is mapped by $T_{1}(c)$ onto a curvilinear segment on $D_{1}(c)$ passing near point $(0,0)$ at the distance of the order $|c|^{l}, l \geq 1$. The same holds true for a symmetric curve on $D_{2}(c)$ being the pre-image w.r.t. $T_{2}(c)$ of the segment of $w_{s}(c)$ (i.e. $u=0)$. Let us cut out on $D_{1}(c), D_{2}(c)$ small disks of the radius of an order $O(\sqrt{|c|})$ centered at points $(0,0)$. Since the map $T(c)$ preserves $\eta$, we cut out thereby by one interval on each curvilinear segment of each disk. After cutting out two remaining segments stay on each disk. Consider the images of the remaining segments on $D_{2}(c)$ under the map $T_{2}(c) \circ T(c)$.

Theorem 5. For $|c|$ small enough the image of each remaining segment is a finite spiral which intersects transversely at a finite number of points the curve $u=0$ in $\Pi^{s}(c)$.

Proof. We follow the lines of the case $c=0$. The difference is that we first cut out the disk on $D_{1}(c)$ of the radius $O(\sqrt{|c|}), \eta \leq \eta(c)$, with the center at $(0,0)$. The image of the segment $v=0$ on $\Pi^{u}(c)$ under the action of the map $T_{1}(c)$ is a smooth curve passing at the distance of the order $c^{l}, l \geq 1$, from the point $(0,0)$ (the case when this curve passes through the point 
$(0,0)$ is not excluded). Therefore the circle of the radius $\eta=\eta(c) \sim|c|$ intersects this curve at two points, i.e. parts of this curve lying outside of the circle are two smooth segments and their images w.r.t. $T(c)$ are two finite spirals which intersect transversely the $T_{2}(c)$-pre-image of the segment $u=0$ from $\Pi^{s}(c)$. Thus, we get a finite number of transverse homoclinic orbits for $\gamma_{c}$. Obviously, the less $|c|$, the more number of transverse homoclinic orbits can be found.

To prove the existence of elliptic points in some neighborhood of the connection in the whole $M$, we first find a countable set of values $c_{n}<0$, for which the system in the level $V_{c_{n}}$ has a non-transverse homoclinic orbit with quadratic tangency for periodic orbit $\gamma_{c_{n}}$. This allows one to apply results on the existence of cascades of elliptic periodic orbits on the levels close to $V_{c_{n}}$ (see, for instance, [15, 16]).

Theorem 6. Suppose inequality $a_{1}^{\prime}(0) \neq 0$ to hold. There is a sequence $c_{n} \rightarrow-0$ such that in the level $V_{c_{n}}$ periodic orbit $\gamma_{c_{n}}$ possesses a homoclinic orbit along which stable and unstable manifolds $W^{s}\left(\gamma_{c_{n}}\right), W^{u}\left(\gamma_{c_{n}}\right)$ have a quadratic tangency. For every such $c_{n}$ there exists a countable set of c-intervals $I_{n m}, I_{n m} \rightarrow c_{n}$ as $m \rightarrow \infty$, whose values $c \in I_{n m}$ represent levels where the system has a 1-round elliptic periodic orbit in this level of the Hamiltonian.

Proof. The inequality $a_{1}^{\prime}(0) \neq 0$ is of the general position condition. It is the analog of the condition $\mathrm{C}$ in [15]. This guarantees that for $|c|$ small enough the $T_{2}(c)$-image of the point $(0,0)$ in $\Pi^{s}(c)$ is an analytic curve intersecting transversely stable manifold $u=0$ of the saddle fixed point. To be precise, we remind we assume coordinates $(u, v)$ not depending on $c$ only maps do. As a corollary of this inequality, by reversibility, there is a $c_{0}<0$ such that for $c \in\left(c_{0}, 0\right)$ the $T_{1}(c)$-image of the segment $v=0$ is a smooth curve that does not pass through the point $(0,0)$ on $D_{1}(c)$ and the distance from $(0,0)$ to this curve is of the order $|c|$.

To prove the first assertion of the theorem, we consider level $V_{c}$ for small negative $c$ and find the image of the segment $v=0$ from $\Pi^{u}(c)$ under the map $T(c) \circ T_{1}(c)$. This is an analytic curve in $D_{2}(c)$. One needs to show that this curve for a countable set of $c$-values touches the $T_{2}(c)$-pre-image of the segment $u=0$ from $\Pi^{s}(c)$.

Let us write down the representation of the map $T(c)$. It is similar to (5) but for $c<0$ function $\Delta_{c}(\eta)$ is analytic and has the form

$$
\Delta_{c}(\eta)=a_{c}^{\prime}(\eta) \ln \frac{d^{2}}{a_{c}(\eta)}, a_{c}(\eta)=-c+\omega \eta+O_{2}(c, \eta)>0 .
$$

The positivity of the function $a_{c}(\eta)$ implies the map $T(c)$ be a local analytic symplectic diffeomorphism in some neighborhood of the point $\left(x_{2}, y_{2}\right)=(0,0)$ for all sufficiently small in modulus negative $c$.

The map $T_{1}(c)$ is also analytic, hence the $T_{1}(c)$-image of the segment $v=0,\left|u-u_{-}\right| \leq \varepsilon$ be an analytic curvilinear segment in $D_{1}(c)$ passing near point $\left(x_{2}, y_{2}\right)=(0,0)$ at the distance of the order $|c|$. This follows from the genericity assumption $a_{1}^{\prime}(0) \neq 0$ and symmetry of $T_{1}(c)$ and $T_{2}(c)$. By symmetry, the $T_{2}(c)$-pre-image of the segment $u=0,\left|v-v_{+}\right| \leq \varepsilon$ is also an analytic curvilinear segment in $D_{2}(c)$ being symmetric w.r.t. $L$ to the segment in $D_{1}(c)$ and passing near the point $\left(\bar{x}_{2}, \bar{y}_{2}\right)=(0,0)$ at the same distance of the order $|c|$.

In polar coordinates on disks $D_{1}(c), D_{2}(c)$ the map $T_{c}$ has of the form

$$
\bar{\eta}=\eta, \quad \bar{\theta}=\varphi+\Delta_{c}(\eta),
$$

with $\Delta_{c}(\eta)=(\omega+\cdots) \ln \left[d^{2} /(-c+\omega \eta+\cdots)\right]$. 
Expanding in formulas for $T_{1}(c)$ coefficients by the Taylor formula up to the terms of the first order in $c$ we get $a(c)=a c+\ldots, a \neq 0, b(c)=b c+\ldots$ Then one has

$$
\Delta_{c}(\eta)=-\omega \ln \frac{d^{2}}{-c+\omega\left[\left(a c+\alpha\left(u-u_{-}\right)\right)^{2}+\left(b c+\gamma\left(u-u_{-}\right)\right)^{2}\right] / 2}+O_{2}(c, \eta) .
$$

On the disk $D_{1}(c)$ the curvilinear segment under consideration is an analytic smooth curve at the distance of the order $|c|$ from $(0,0)$, so there is a circle $\eta=\eta_{c}$ such that this circle and the curve have a common point and they are tangent at this point. In principle, this point can be not unique. Other points of this curve are outside of this circle.

Local map $T(c)$ preserves $\eta$, hence the $T(c)$-image on $D_{2}(c)$ of the curve is a spiral-shape curve that lies outside of the circle $\eta=\eta_{c}$ on $D_{2}(c)$. By symmetry, on the same circle on $D_{2}(c)$ there are other its points of tangency with the curve being $T_{2}(c)$-pre-image of the segment $u=0$ from $\Pi^{s}(c)$. An important observation is the following assertion.

Lemma 2. For $c$ small enough the only point of tangency the circle and the curve on $D_{1}(c)$ exists. The tangency at this point is quadratic.

Proof. Denote $\sigma_{c}^{s}, \sigma_{c}^{u}$ circles $\eta=\eta_{c}$ on $D_{1}(c), D_{2}(c)$, respectively. By symmetry, it is sufficient to prove the assertion for the closed curve $T_{2}(c)\left(\sigma_{c}^{u}\right)$, i.e. this curve is quadratically tangent to $u=0$ at exactly one point as $|c|$ small enough. The circle $\sigma_{c}^{u}$ has the representation in polar coordinates $\bar{x}_{2}=\sqrt{2 \eta_{c}} \cos \theta, \bar{y}_{2}=\sqrt{2 \eta_{c}} \sin \theta, r(c)=\sqrt{2 \eta_{c}} \sim|c|$. Thus, its $T_{2}(c)$-image is (4)

$u_{1}=a_{1}(c)+r(c)[\gamma(c) \cos \theta+\alpha(c) \sin \theta+O(r)], v_{1}-v_{+}=b_{1}(c)-r(c)[\delta(c) \cos \theta+\beta(c) \sin \theta+O(r)]$.

First we find the points where a tangent to this curve is collinear with the vector $(0,1)$, i.e. $u_{1}^{\prime}(\theta)=0$. This gives the equation $-\gamma \sin \theta+\alpha \cos \theta+O(r)=0$. It has two roots defined up to $O(r)$ as $\theta_{1}=\rho, \theta_{2}=\rho+\pi$, where $\sin \rho=\alpha / \sqrt{\alpha^{2}+\gamma^{2}}, \cos \rho=\gamma / \sqrt{\alpha^{2}+\gamma^{2}}$. Equating $u_{1}\left(\theta_{i}\right)=0$ we come to the relations relative $r: r(c)= \pm a_{1}(c) / \sqrt{\alpha^{2}+\gamma^{2}}+O\left(c^{2}\right)$, where the sign is determined by that $\theta_{i}$ for which $r(c)>0$. Due to assumption $a_{1}^{\prime}(0) \neq 0$, we get a unique root providing the tangency of an even order.

To prove the tangency be quadratic, one needs to check that for $c$ small enough the derivative $u_{1}^{\prime \prime}\left(v_{1}\right) \neq 0$ at the tangency point. This derivative in the parametric form is given as (we omit subscript 1 in this calculation)

$$
u^{\prime \prime}(v)=\frac{u_{\theta}^{\prime \prime} v_{\theta}^{\prime}-u_{\theta}^{\prime} v_{\theta}^{\prime \prime}}{v_{\theta}^{\prime 3}}=\sqrt{\alpha^{2}+\gamma^{2}} \frac{[-(\alpha \delta-\beta \gamma)+O(r)]}{ \pm r(1+O(r))}= \pm \sqrt{\alpha^{2}+\gamma^{2}} r(c)^{-1}(1+O(r(c))) .
$$

Since we saw that $r(c) \sim|c|$ as $|c| \rightarrow 0$, this derivative is as larger in modulus as smaller $|c|$ is. Thus, we conclude the tangency be quadratic.

So, we have on $D_{2}(c)$ two analytic curves: a spiral and a curve, both they touch the circle $\eta=\eta_{c}$ at only points (generally speaking, different ones). Now let us follow a mutual position of these two point on the circle as $c \rightarrow-0$. The point on the curve tends to the point $(0,0)$ as $c \rightarrow-0$ with a definite tangent. But the tangency point of the spiral, as we shall prove below, rotates monotonically as $c \rightarrow-0$ performing infinitely many full revolutions in the angle. This implies that the point of tangency for the spiral infinitely many times $c_{n}$ pass through the point of tangency for the curve giving quadratic tangency of he spiral and the curve (see, Fig. 9). 
Let us call the unique point of tangency of the circle and the spiral on $D_{2}(c)$ a nose of the spiral. Near this point, due to a quadratic tangency, the spiral is located out of the disk bounded by the circle. Let us show that the nose of the spiral moves monotonically in $\theta$ as $c \rightarrow-0$.

The coordinates of the nose correspond to that point of the segment $v=0$ where the $T_{1}(c)$ pre-image of the circle $\eta=\eta_{c}$ in $D_{1}(c)$ touches the segment. The angle $\theta(c)$ corresponding to the nose of the spiral is calculated using the formula $\theta(c)=\varphi(c)+\Delta_{c}\left(\eta_{c}\right)$ where the values $(\varphi(c), \eta(c))$ have to be inserted. As we saw when proving the lemma 2, the angle $\varphi(c)$ has a definite limit as $c \rightarrow-0$, since the point of tangency of the curvilinear segment and the circle $\eta=\eta_{c}$ on $D_{1}(c)$ and the point of tangency of the circle $\eta=\eta_{c}$ and a curvilinear segment on $D_{2}(c)$ are connected by the symmetry relation $L:\left(x_{2}, y_{2}\right) \rightarrow\left(\bar{x}_{2}, \bar{y}_{2}\right) \bar{x}_{2}=-x_{2}, \bar{y}_{2}=y_{2}$. We shall show that the value $\Delta_{c}\left(\eta_{c}\right)$ tends monotonically to infinity as $c \rightarrow-0$. If so, this gives necessary conclusion on the infinite number of full revolutions in the angle $\theta$.

The value $\eta_{c}$ at the tangency point is equal to

$$
\eta_{c}=\frac{1}{2}\left(x_{2}^{2}(c)+y_{2}^{2}(c)\right)=r^{2}(c) / 2=\frac{a_{1}^{2}(c)}{2\left(\alpha^{2}(c)+\gamma^{2}(c)\right)}+O\left(c^{3}\right) .
$$

Therefore, we have

$$
\Delta_{c}\left(\eta_{c}\right)=\left(\omega+O\left(c^{2}\right)\right) \ln \frac{d^{2}}{-c+\omega \frac{1}{2\left(\alpha^{2}+\gamma^{2}\right)} a_{1}^{2}(c)+O\left(c^{3}\right)} \sim-\ln (-c) .
$$

Thus, $\theta(c)$ depends on $c$ monotonically and increases unboundedly as $c \rightarrow-0$. Therefore the point in $D_{2}(c)$, being the nose of the spiral, infinitely many times $c_{n}$ coincides with the point on the same circle where the pre-image of the segment $u=0$ from $\Pi^{s}(c)$ touches the circle.

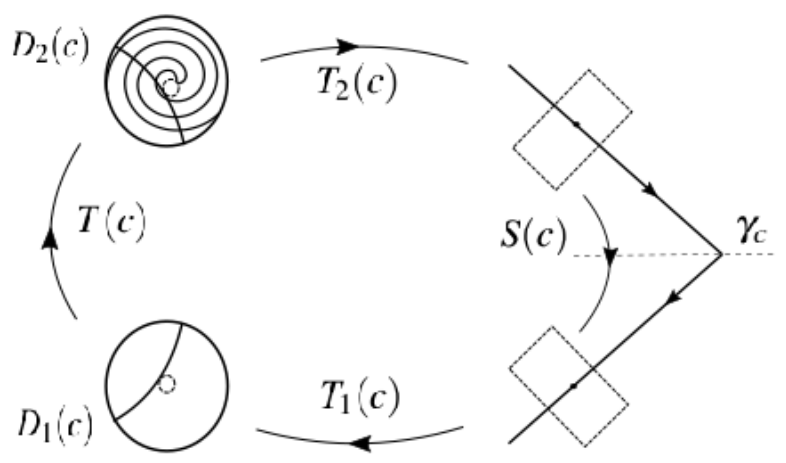

Figure 9: Case 1: Poincaré map as $c<0$, a mechanism of homoclinic tangency.

The second assertion of the theorem follows from the theorem on the existence of elliptic points near a homoclinic tangency for a symplectic map (see, for instance, [15, 16]).

Theorem 7. Let $f$ be a smooth (at least $C^{4}$ ) symplectic map having a saddle fixed point $p$ and a homoclinic orbit $f^{n}(q)$ through the point $q \neq p$. Suppose stable and unstable curves of $p$ are quadratically tangent at $q$. Then for any generic smooth one-parametric family of smooth symplectic maps $f_{\mu}$ that coincides as $\mu=0$ with $f$ on any segment $\left[-\mu_{0}, \mu_{0}\right]$ there is an integer $k_{0} \in \mathbb{Z}$ and infinitely many open intervals $I_{k}, k \geq k_{0}$, such that $I_{k} \rightarrow 0$, as $k \rightarrow \infty$, and the map $f_{\mu}, \quad \mu \in I_{k}$ has a one-round elliptic homoclinic orbit (of the period $q+k$ ). 


\section{Hyperbolicity and ellipticity as $c>0$}

As we know, any level $V_{c}$ for $c>0$ small enough contains a Lyapunov saddle periodic orbit $l_{c}$ (remind that we assume $\omega>0$ ). Its local stable $W^{s}\left(l_{c}\right)$ and unstable $W^{u}\left(l_{c}\right)$ manifolds belong to $V_{c}$ and their extension by the flow occur near $W^{s}(p)$ and $W^{u}(p)$, respectively. Therefore, they intersect cross-sections $D_{1}(c)$ and $D_{2}(c)$ along the circles $\sigma_{s}(c), \sigma_{u}(c)$.

As was indicated above, all flow orbits cutting $D_{1}(c)$ inside of the circle $\sigma_{s}(c)$ go out from $U$ not intersecting $D_{2}(c)$. That is why, we do not track for these orbits. But flow orbits cutting $D_{1}(c)$ outside of $\sigma_{s}(c)$, as time increases, do intersect $D_{2}(c)$ and further the cross-section $\Sigma_{c}$. We construct here a hyperbolic set that is formed near a heteroclinic connection that involve a pair of saddle periodic orbits $l_{c}, \gamma_{c}$ and four transverse heteroclinic orbits, of which two go, as time increases, from $l_{c}$ to $\gamma_{c}\left(\right.$ near $\left.\Gamma_{2}\right)$, and two others - from $\gamma_{c}$ to $l_{c}\left(\right.$ near $\left.\Gamma_{1}\right)$. Besides, there is a countable set of transverse homoclinic orbits for every periodic orbits $l_{c}$ and $\gamma_{c}$. All this the base for constructing the hyperbolic set.

Theorem 8. For $c>0$ small enough $W^{s}\left(l_{c}\right)$ and $W^{u}\left(\gamma_{c}\right)$ intersect transversely each other along two heteroclinic orbits $\Gamma_{11}(c), \Gamma_{12}(c)$ and, by symmetry, $W^{u}\left(l_{c}\right)$ and $W^{s}\left(\gamma_{c}\right)$ intersect transversely each other along two heteroclinic orbits $\Gamma_{21}(c)=L\left(\Gamma_{11}(c)\right), \Gamma_{22}(c)=L\left(\Gamma_{12}(c)\right)$, forming thereby a transverse heteroclinic connection.

The trace of $W^{u}\left(\gamma_{c}\right)$ in $\Pi^{s}(c)$ (the image of the segment $v=0$ under the action of the map $\left.T_{2}(c) \circ T(c) \circ T_{1}(c)\right)$ consists of a pair of spiral-shape analytic curves which wind both on the closed curve $T_{2}(c)\left(\sigma_{u}(c)\right)$ and intersect transversely the segment $u=0$ at a countable set of points being traces of transverse Poincaré homoclinic orbits of the periodic orbit $\gamma_{c}$.

By Smale's $\lambda$-lemma, for $n$ large enough, the closed curve $T_{2}(c)\left(\sigma_{u}(c)\right)$ contains two its segments with end points on the segment $u=0$, whose $n$-iterations under map $S(c)$ give a countable family of analytic curves smoothly accumulating, as $n \rightarrow \infty$, to the segment $v=0$. There is an integer $n_{0}(s)$ such that for $n>n_{0}(s)$ these curves transversely intersect the closed curve $T_{1}^{-1}(c)\left(\sigma_{s}(c)\right)$ giving a countable set of points being traces of transverse homoclinic orbits for $l_{c}$ (see, Fig. 10).

Proof. Consider first $T_{2}(c)$-image of the circle $\sigma_{u}(c)$ on the disk $\Sigma_{c}$. Recall that radius of the circle $\sigma_{u}(c)$ is of the order $\sqrt{c}$, since it defined by the root of the equation $a_{c}(\eta)=-c+\omega \eta+O_{2}(c, \eta)=0$. Hence, we get $\eta(c)=c / \omega+O\left(c^{2}\right)$. On the other hand, due to an analytic dependence of $T_{2}(c)$ in $c$, the $T_{2}(c)$-image of the center $(0,0)$ analytically depends in $c$. Therefore, the distance from the point $(0,0)$ to the curve being the $T_{2}(c)$-pre-image of the segment $u=0$ in $\Pi^{s}(c)$ has the order $c^{l}, l \geq 1$. Moreover, $l=1$ if the inequality $a_{1}^{\prime}(0) \neq 0$ holds (see above). This implies that the curve $T_{2}(c)\left(\sigma_{u}(c)\right)$ intersects, for $c$ small enough, segment $u=0$ transversely at two points. Indeed, the curve $T_{2}(c)\left(\sigma_{u}(c)\right)$ can be written in a parametric form with parameter $\theta$ as

$u=a_{1}(c)+r(c)[\gamma(c) \cos \theta+\alpha(c) \sin \theta+O(r)], v-v_{+}=b_{1}(c)-r(c)[\delta(c) \cos \theta+\beta(c) \sin \theta+O(r)]$,

where $r(c)=\sqrt{2 \eta(c)}=\sqrt{c / \omega+O\left(c^{2}\right)}$. Equating $u_{1}=0$ to find intersection points with segment $u=0$ and dividing both sides at $r(c) \sqrt{\alpha^{2}+\gamma^{2}}$ we come to the equation w.r.t. $\theta$

$$
A(c)+\cos (\theta-\rho)+O(c)=0, A(c)=a_{1}(c) / r(c) \sim \sqrt{c},
$$

that has two simple roots for $c$ small enough. These simple roots correspond to two transverse intersection points. The flow orbits through these points are just $\Gamma_{21}(c), \Gamma_{22}(c)$. By the reversibility of the map, the closed curve $T_{1}^{-1}(c)\left(\sigma_{s}(c)\right)$ intersects transversely at two points the 
segment $v=0$ in $\Pi^{u}(c)$ as well. The flow orbits through these intersection points are $\Gamma_{11}(c)$, $\Gamma_{12}(c)$.

Now consider the curvilinear segment being the $T_{1}(c)$-image of $v=0$ in $D_{1}(c)$. As was proved, this curve intersect transversely at two points the circle $\sigma_{s}(c)$ which divide the curve into three pieces. The flow orbits, passing through the middle piece, leave the neighborhood of the connection, but two remaining pieces give two analytic curves whose $T(c)$-images are two infinite spirals on $D_{2}(c)$ which wind up the circle $\sigma_{u}(c)$. Their $T_{2}(c)$-images gives two countable families of transverse homoclinic orbits for $\gamma_{c}$.

In order to find transverse homoclinic orbits to $l_{c}$, we remark that the segment in $\Pi^{s}(c)$ given as $u=\kappa>0$ for $\kappa$ small enough intersect the closed curve $T_{2}(c)\left(\sigma_{u}(c)\right)$ transversely at two points. The same holds true for all pieces of $T_{2}(c)$-images of both spirals winding up at $\sigma_{u}(c)$ in $D_{2}(c)$. Thus we have two countable families of curvilinear segments smoothly accumulating to two segments of the curve $T_{2}(c)\left(\sigma_{u}(c)\right)$. By Smale's $\lambda$-lemma [41], there is an integer $n_{0}>0$ such that all $S^{n}(c)$-images of curves of both countable families intersect transversely the closed curve $T^{-1}(c)\left(\sigma_{s}(c)\right.$ in $\Pi^{u}(c)$. Thus, we have an invariant hyperbolic set in each level $V_{c}, c>0$ small enough.

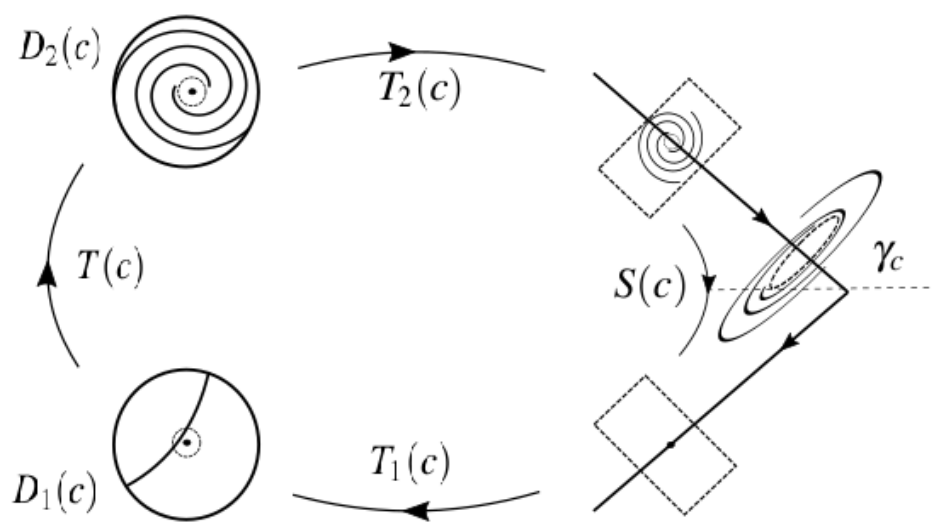

Figure 10: Poincaré map as $c>0$, hyperbolic set and tangency.

The hyperbolic set, we have constructed, does not exhaust invariant sets in the level $V_{c}$. One can mention the wild hyperbolic sets existing near the quadratic homoclinic tangencies [3]. Here we only prove the existence of elliptic periodic orbits for intervals of $c$-values accumulating at $c=0$. To that end, we first prove

Theorem 9. There is a sequence of $c_{n} \rightarrow 0$ such that in the level $V_{c_{n}}$ the Lyapunov saddle periodic orbit $l_{c_{n}}$ possesses a homoclinic orbit along which $W^{s}\left(l_{c_{n}}\right)$ and $W^{u}\left(l_{c_{n}}\right)$ have quadratic tangency.

Proof. As the system is reversible and the related Poincaré map is also reversible, it is sufficient to prove that there exist $c_{n}$ such that the trace on $\Sigma_{n}$ of $W^{u}\left(l_{c_{n}}\right)$ is a convex closed curve being quadratically tangent to the line of $F i x(L)$ - the diagonal $u=v$.

As was proved above, for $c_{0}$ sufficiently small as $0<c \leq c_{0}$, the trace of $W^{u}\left(l_{c}\right)$ in $\Pi^{s}(c)$ is a closed curve which intersects the segment $u=0$ at two points. The $S(c)$-pre-images of the line $u=v$ are a sequence of analytic curvilinear segments, given as $u=u_{k}(v, c)$, which tend in 
$\Pi^{s}(c)$ to $u=0$ in $C^{2}$-topology uniformly w.r.t. $c$, as $k \rightarrow \infty$. Indeed, the inverse iterations of $S$ are given as $u_{-n}=f^{n}(\zeta) u_{-n+1}, v_{-n}=v_{-n+1} / f^{n}(\zeta), \zeta=u_{0} v_{0}=u_{-1} v_{-1}=\cdots=u_{-n} v_{-n}$. The function $u_{-n}=g_{n}\left(v_{-n}\right)$ is found as a solution w.r.t. $u$ of the equation $u=v f^{2 n}(u v)$. Multiplying both sides on $v$, we get the equation $\zeta=v^{2} f^{2 n}(\zeta)$. Due to form of $f(\zeta)=\nu+O_{1}(\zeta)$ we have an estimate $|f| \leq(1+\nu) / 2<1$ for sufficiently small $|\zeta|$. Thus, for any fixed $v,\left|v-v_{+}\right| \leq \delta$ we find the unique solution of the equation $\zeta_{n}(v)$. This function is analytic and family tends uniformly to zero as $n \rightarrow \infty$. This give functions $u_{n}(v, c)=v f^{2 n}\left(\zeta_{n}(v)\right)$. This family tends to zero as $[(1+\nu) / 2]^{2 n}$. Since they approach to zero in $C^{2}$-topology (in fact, in any $C^{k}, k \geq 2$ ), this implies that for $n$ large enough the intersection of the graph of function $u_{n}(v, c)$ with the closed curve $T_{2}(c)\left(\sigma_{u}(c)\right.$ occurs in two points similar as for $u=0$.

Fix some $0<c \leq c_{0}$. For $c_{0}$ is small enough, the closed curve is, up to third order terms, an ellipse in $\Pi^{s}(c)$ whose center approach to the line $u=0$ with the order $c$ and its principal axes have lengths of the order $\sqrt{c}$ and their rotation angle depends as $c$ and has a limit defined by the matrix of the linearized map $T_{2}(0)=T_{2}$. This implies this family of closed curves intersects, as $c \rightarrow 0$, all graphs of the functions $u_{n}(v, c)$ and this intersection for the individual curve is either transversal or quadratically tangent, or no intersection points at all. Those values of $c$ when the related curve of the family is tangent to a fixed $u_{n}(v, c)$ give the values $c_{n}$ we search for.

Now we can again to apply the Theorem 7 on the existence of elliptic periodic point in a generic one-parameter unfolding of two-dimensional symplectic diffeomorphisms that contains a diffeomorphism with a quadratic homoclinic tangency [15, 16]).

\section{The case $2, c>0$}

As was shown above, for the case 2 all orbits in the level $V_{0}$ passing through a small neighborhood of the connection, other than those of the connection itself, leave this neighborhood and no orbits exist which stay forever in this neighborhood. Levels $V_{c}, c<0$, contain no orbits at all which stay wholly in these levels, since orbits entering to the solid cylinder through $D_{1}(c)$, exit from the neighborhood of the point $p$ without intersecting $D_{2}(c)$. That is why we consider levels $V_{c}$ for $c>0$, where orbits arise lying wholly in a neighborhood of the connection. On the corresponding transversal disk $D_{1}(c)$ these orbits enter to the solid cylinder through points lying inside the circle $\sigma_{s}(c)$ and they exit through $D_{2}(c)$ inside of the circle $\sigma_{u}(c)$ from another solid cylinder (see, Remark 6).

Consider the image w.r.t. the map $T_{2}(c) \circ T(c) \circ T_{1}(c)$ of the trace of the unstable manifold $W^{u}\left(\gamma_{c}\right)$ (i.e. the segment $v=0$ from $\Pi^{u}(c)$ ). As was discussed above, generally $T_{1}(c)$-image of this segment on the disk $D_{1}(c)$ is an analytic curvilinear segment whose distance from the center of the disk $(0,0)$ has the order $c^{l}, l \geq 1$, due to the analytic dependence of the map $T_{1}(c)$ in $c$. If the genericity assumption above $a_{1}^{\prime}(0)$ holds, then $l=1$. On the disk $D_{1}(c)$ there is a circle $\sigma_{s}(c)$ defined as $\eta=\eta(c)=c / \omega+O\left(c^{2}\right)$, being the trace of the stable manifold $W^{s}\left(l_{c}\right)$. Thus, its radius is of the order $\sim \sqrt{c}$. This implies, as above, that $\sigma_{s}(c)$ and the curvilinear segment (trace of $W^{s}\left(\gamma_{c}\right)$ ) intersect each other transversely at two points for $c$ small enough.

Consider now that interval of the curvilinear segment which lies on $D_{1}(c)$ inside of the circle $\sigma_{s}(c)$. Keeping in mind the modification of the formula for $\Delta_{c}(\eta)$ (see, Remark 6), we see that this interval (without its two extreme points on the circle $\sigma_{s}(c)$ ) is transformed by the map $T(c)$ on $D_{2}(c)$ where it forms an infinite spiralling analytic curve that winds up by its both ends on the circle $\sigma_{u}(c)$ (see, Fig. 11). On the same disk $D_{2}(c)$ there is an analytic curvilinear 
segment being the $T_{2}(c)$-pre-image of the segment $u=0$ from $\Pi^{s}(c)$. The curvilinear segment intersects transversely the circle $\sigma_{u}(c)$, this follows from its symmetry with the related curve in $D_{1}(c)$. Since the double spiral winds up by its both ends on the circle $\sigma_{s}(c)$ and the segment is transverse to the circle, we get, as above, a countable set of intersection points through which transverse homoclinic orbits of $\gamma_{c}$ pass.

Here we also have a countable set of intervals of $c$ on which elliptic periodic orbits exist in $V_{c}$. Their proof is done by exactly the same manner as for the case 1 and $c>0$. The crucial point here is again to find a sequence of $c_{n} \rightarrow 0$ such that in $V_{c_{n}}$ a tangent symmetric homoclinic orbit of $l_{c}$ exists. We again iterate by the maps $S^{n}(c)$ on the disk $\Sigma(c)$ the closed curve $T_{2}(c)\left(\sigma_{u}(c)\right)$ and find its tangency with the line $u=v$ of the trace Fix $(L)$. The consideration is the same as in the preceding section. Thus, we obtain

Theorem 10. For the case 2 there exists $c_{0}>0$ small enough such that on the interval $\left(0, c_{0}\right)$ a countable set of intervals exists whose values of c correspond to levels $V_{c}$ containing 1-round elliptic periodic orbit in a four-dimensional neighborhood of the initial heteroclinic connection.

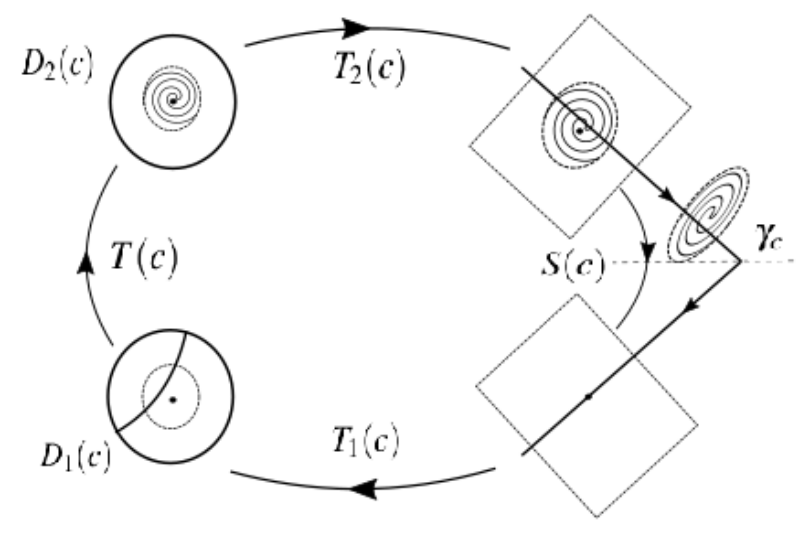

Figure 11: Case 2: Poincaré map as $c>0$, hyperbolic set and tangency.

\section{1-parameter family of reversible systems: homoclinics of the saddle-center}

We consider in this section a generic 1-parameter family of reversible Hamiltonian systems $X_{H_{\mu}}$ being an unfolding of a system that has at $\mu=0$ a heteroclinic connection studied in Sections above. The main result here is a theorem on the existence of a countable set of parameter values $\mu$ accumulating to $\mu=0$ for which the related system has a homoclinic orbit of the saddle-center. Here also it will be shown that emerging homoclinic orbits of the saddle-center satisfy the general position conditions found in [22, 20]. These conditions guarantee the existence of complicated dynamics in the system and its non-integrability [21. It is worth emphasizing that the result does not depend on what type of the connection is, the first or second one.

Recall that in the class of $C^{2}$-smooth Hamiltonian systems existence of a saddle-center equilibrium is a generic phenomenon. Existence of a saddle periodic orbit is also a generic phenomenon. But for general reversible perturbations heteroclinic orbits joining a saddle-center and a saddleperiodic orbit can be destroyed. We want to prove that generically here homoclinic orbits to 
a perturbed saddle-center emerge. The theorem we prove can serve a criterion of the existence of homoclinic orbits of the saddle-center. It is worth remarking that finding such orbits is a rather delicate problem generally. Such a theorem can also be used in the case when we deal with a two-parameter family of reversible Hamiltonian systems and for some specific values of the parameters the system has a connection mentioned above. Then we can find a countable set of curves in the parameter space such that for parameters on this curve the system has a homoclinic orbit of the saddle-center.

Theorem 11. Let $X_{H_{\mu}}$ be a generic one-parameter family of reversible analytic Hamiltonian systems and at $\mu=0$ the system has a heteroclinic connection of the type 1 or 2 studied above. Then there exists a sequence of parameter values $\mu_{n}$ accumulating to $\mu=0$ such that the related system of the family has a homoclinic orbit of the general type of a saddle-center. Related values $\mu_{n}$ have the same sign.

Proof. Because of the reversibility, it is sufficient to prove the existence of a sequence $\mu_{n} \rightarrow 0$ for which the Hamiltonian system $X_{H_{\mu_{n}}}$ has an unstable separatrix of the saddle-center that intersects the cross-section $\Sigma\left(\mu_{n}\right)$ at the line Fix $(L)$.

Remind that for the case of a smooth one-parameter family of reversible analytic Hamiltonian systems being an unfolding of a system with a heteroclinic connection, all objects under consideration, a saddle-center, a periodic orbit in the singular level of the Hamiltonian, their stable and unstable manifolds smoothly depend on $\mu$. As was indicated above, Moser theorems hold also for systems depending on parameters, therefore there is a smoothly depending on a parameter change of variables such that in new coordinates $\left(x_{1}, y_{1}, x_{2}, y_{2}\right)$ in a neighborhood of equilibrium $p_{\mu}$ the Hamiltonian has the form of the analytic functions $h_{\mu}$ in variables $\xi=x_{1} y_{1}, \eta=\left(x_{2}^{2}+y_{2}^{2}\right) / 2$. The difference with the case without parameters is in a smooth dependence on the parameter $\mu$ of coefficients of the function $h_{\mu}$. Further we work in these coordinates, thus cross-sections $D_{1}$ и $D_{2}$ to separatrices in the singular level of the Hamiltonian and $N$ can be regarded fixed and not depending on the parameter. But in global maps zero order terms smoothly depending on $\mu$ appear, as separatrices of the perturbed saddle-center do not lie, generally speaking, on manifolds of the saddle periodic orbit.

The form of the perturbed global maps is as follows

$$
\begin{aligned}
& T_{2}(\mu): u_{1}=a(\mu)+\gamma(\mu) \bar{x}_{2}+\alpha(\mu) \bar{y}_{2}+\cdots \\
& v_{1}-v_{+}=b(\mu)-\delta(\mu) \bar{x}_{2}-\beta(\mu) \bar{y}_{2}+\cdots \\
& T_{1}(\mu): x_{2}=b(\mu)+\alpha(\mu)\left(u-u_{-}\right)+\beta(\mu) v+\cdots \\
& \quad y_{2}=a(\mu)+\gamma(\mu)\left(u-u_{-}\right)+\delta(\mu) v+\cdots, \\
& S(\mu): \quad u_{1}=u / f_{\mu}(\zeta), v_{1}=v f_{\mu}(\zeta), \zeta=u v, f_{\mu}=\nu(\mu)+O_{1}(\zeta) .
\end{aligned}
$$

The genericity condition for the family in coordinates means the inequality to hold $a^{\prime}(0) \neq 0$. In virtue of the assumptions on the family we have $a(0)=0, b(0)=0, \nu(0)=\nu<1$. Geometrically, the genericity condition means that for $\mu \neq 0$ the trace of unstable separatrix of the saddlecenter intersects the trace of the stable manifold of the saddle periodic orbit transversely as $\mu$ varies. More spectacularly, this can be imagined in the space $(u, v, \mu)$ where the segment $(0,0, \mu)$ represents the 1 -parameter family of saddle fixed points of the maps, the rectangular $u=0$ represents the family of traces of stable manifolds of fixed points, and $v=0$ is a family of traces of unstable manifolds of the saddle fixed points. The curve of traces on the crosssection of unstable separatrices of the saddle-centers for every small $\mu$ intersects transversely the rectangular $u=0$ at a unique point where $\mu=0$. 
Consider now the point $(0,0)$ on the disk $D_{2}$ being the trace of the unstable separatrix of the perturbed saddle-center. This point under the action of the map $\left.S_{(}^{n} \mu\right) \circ T_{2}(\mu)$ transforms into $u_{n}=a(\mu) / f_{\mu}^{n}(\zeta), \quad v_{n}=\left(v_{+}+b(\mu)\right) f_{\mu}^{n}(\zeta)$. The condition this point to lie on the line of fixed points of involution gives the equality $u_{n}=v_{n}$, i.e. $a(\mu) / f_{\mu}^{n}(\zeta)=\left(v_{+}+b(\mu)\right) f_{\mu}^{n}(\zeta)$, $\zeta=a(\mu)\left(v_{+}+b(\mu)\right)$. Let us write down this equation w.r.t. $\mu$ in the form

$$
\frac{a(\mu)}{v_{+}+b(\mu)}=f_{\mu}^{2 n}\left(a(\mu)\left(v_{+}+b(\mu)\right)\right)>0
$$

Function $r(\mu)$ in the left side is defined on some neighborhood of $\mu=0, r(0)=0, r^{\prime}(0)=$ $a^{\prime}(0) / v_{+} \neq 0$, so, this function is strictly monotone. The sequence of functions in the right side as $n \rightarrow \infty$ is a sequence of functions defined in a neighborhood of zero and tending uniformly to zero. Thus, for large enough $n$ for those values of $\mu$, where $a(\mu)$ is positive, the equation for every such $n$ has a unique solution $\mu_{n} \rightarrow 0$ as $n \rightarrow \infty$. Asymptotics of values $\mu_{n}$, for which homoclinic orbits of the saddle-center exist is as follows

$$
\mu_{n}=\frac{\nu^{2 n} v_{+}}{a^{\prime}(0)}
$$

We shall now show that for these values $\mu_{n}$ homoclinic orbits of the saddle-center for the related Hamiltonian system satisfy the genericity condition from [22]. In Moser coordinates in a neighborhood of the saddle-center this conditions means that the linearization matrix of the global map at the trace of a homoclinic orbit differs from a rotation matrix.

Consider $\mu=\mu_{n}$. The global map in a neighborhood of the homoclinic orbit is the composition of maps $T_{1}^{\mu} \circ S^{\mu} \circ T_{2}^{\mu}$, i.e.

$$
\begin{gathered}
x_{2}=\left[b(\mu)+\nu^{-n}(\mu) \alpha(\mu) a(\mu)-\alpha(\mu) u_{-}+\beta(\mu) v_{+}+\nu^{n}(\mu) \beta(\mu) b(\mu)+\right. \\
+\left[\nu^{-n}(\mu) \alpha(\mu) \gamma(\mu)-\nu^{n} \beta(\mu) \delta(\mu)\right] \bar{x}_{2}+\left[\nu^{-n}(\mu) \alpha^{2}(\mu)-\nu^{n}(\mu) \beta^{2}(\mu)\right] \bar{y}_{2}+\cdots, \\
y_{2}=\left[a(\mu)+\nu^{-n}(\mu) \gamma(\mu) a(\mu)-\gamma(\mu) u_{-}+\delta(\mu) v_{+}+\nu^{n}(\mu) \delta(\mu) b(\mu)\right]+ \\
+\left[\nu^{-n} \gamma^{2}(\mu)-\nu^{n} \delta^{2}(\mu)\right] \bar{x}_{2}+\left[\nu^{-n}(\mu) \alpha(\mu) \gamma(\mu)-\nu^{n}(\mu) \beta(\mu) \delta(\mu)\right] \bar{y}_{2}+\cdots
\end{gathered}
$$

Zero order terms of $T_{1}^{\mu} \circ S^{\mu} \circ T_{2}^{\mu}$ are equal to zero. Denote $A=\nu^{-n}(\mu) \alpha(\mu) \gamma(\mu)-\nu^{n} \beta(\mu) \delta(\mu), \quad B=$ $\nu^{-n}(\mu) \alpha^{2}(\mu)-\nu^{n}(\mu) \beta^{2}(\mu), \quad C=\nu^{-n} \gamma^{2}(\mu)-\nu^{n} \delta^{2}(\mu), \quad D=\nu^{-n}(\mu) \alpha(\mu) \gamma(\mu)-\nu^{n}(\mu) \beta(\mu) \delta(\mu)$. Then the map $T_{1}^{\mu} \circ S^{\mu} \circ T_{2}^{\mu}$ is written as follows

$$
x_{2}=A \bar{x}_{2}+B \bar{y}_{2}+\ldots, \quad y_{2}=C \bar{x}_{2}+D \bar{y}_{2}+\ldots
$$

Since $T_{1}^{\mu}, S^{\mu}, T_{2}^{\mu}$ are symplectic maps, then their composition $T_{1}^{\mu} \circ S^{\mu} \circ T_{2}^{\mu}$ is also symplectic map. Let us show that the composition $T_{1}^{\mu} \circ S^{\mu} \circ T_{2}^{\mu}$ is different from the a rotation map. To this end, consider circles $S_{1}: x_{2}^{2}+y_{2}^{2}=2 \eta, \quad S_{2}: \bar{x}_{2}^{2}+\bar{y}_{2}^{2}=2 \eta$ of the same radius in neighborhoods in $D_{1}, D_{2}$, respectively, and prove that the circle $S_{2}$ under the action of $T_{1}^{\mu} \circ S^{\mu} \circ T_{2}^{\mu}$ intersects $S_{1}$ at four points.

For this we use symplectic polar coordinates:

$$
\begin{array}{ll}
x_{2}=\sqrt{2 \eta} \cos \theta, & y_{2}=\sqrt{2 \eta} \sin \theta \\
\bar{x}_{2}=\sqrt{2 \eta} \cos \varphi, & \bar{y}_{2}=\sqrt{2 \eta} \sin \varphi
\end{array}
$$


Then the condition of intersection $S_{1}$ and $T_{1}^{\mu} \circ S^{\mu} \circ T_{2}^{\mu}\left(S_{2}\right)$ is equivalent to the equation:

$$
\begin{gathered}
1=(A \cos \varphi+B \sin \varphi)^{2}+(C \cos \varphi+D \sin \varphi)^{2} \Leftrightarrow \\
\frac{A^{2}+B^{2}+C^{2}+D^{2}}{2}+\frac{A^{2}+C^{2}-B^{2}-D^{2}}{2} \cos 2 \varphi+(A B+C D) \sin 2 \varphi=1 \Leftrightarrow \\
\sin (2 \varphi+F)=\frac{2-\left(A^{2}+B^{2}+C^{2}+D^{2}\right)}{\sqrt{4(A B+C D)^{2}+\left(A^{2}+C^{2}-B^{2}-D^{2}\right)^{2}}}
\end{gathered}
$$

The equation (8) has four solutions on the segment $[0,2 \pi)$, if the inequality holds

$$
\left(2-\left(A^{2}+B^{2}+C^{2}+D^{2}\right)\right)^{2}<4(A B+C D)^{2}+\left(A^{2}+C^{2}-B^{2}-D^{2}\right)^{2} .
$$

Taking into account that $A=D, \quad A D-B C=1$ we get $2-2 A^{2}-B^{2}-^{2}<0$. Insert into the last inequality $A, B, C \quad 2-2\left(\nu^{-n} \alpha(\mu) \gamma(\mu)-\nu^{n} \beta(\mu) \delta(\mu)\right)^{2}-\left(\nu^{-n}(\mu) \alpha^{2}(\mu)-\nu^{n}(\mu) \beta^{2}(\mu)\right)^{2}-$ $\left(\nu^{-n} \gamma^{2}(\mu)-\nu^{n} \delta^{2}(\mu)\right)^{2}<0$. After some calculations we get $2-\left(\alpha^{2}(\mu)+\gamma^{2}(\mu)\right)^{2} \nu^{-2 n}(\mu)+$ $2\left(\alpha^{2}(\mu) \beta^{2}(\mu)-\gamma^{2}(\mu) \delta^{2}(\mu)\right)^{2}-\left(\beta^{2}(\mu)+\delta^{2}(\mu)\right)^{2} \nu^{2 n}(\mu)<0$. The last inequality will hold beginning since some $n>n_{0}$ as $\left(\beta^{2}(\mu)+\delta^{2}(\mu)\right)^{2} \nu^{2 n}(\mu) \rightarrow 0, \quad\left(\alpha^{2}(\mu)+\gamma^{2}(\mu)\right)^{2} \nu^{-2 n}(\mu) \rightarrow \infty$ as $n \rightarrow+\infty$. Thus we have proved the map $T_{1} \circ S \circ T_{2}$ have its linear part different from the rotation matrix.

\section{Conclusion}

In the paper we study some dynamical phenomena in a one-parameter unfolding reversible Hamiltonian systems which contain a system with a symmetric heteroclinic connection that involves a symmetric saddle-center, a symmetric saddle periodic orbit in the same level of the Hamiltonian and a pair of heteroclinic orbits joining the saddle-center and periodic orbit and permutable by the reversible involution. We found several types of hyperbolic sets, cascades of elliptic periodic orbits and countable sets of parameters for which homoclinic orbits to the saddle-center exist. All this characterized the chaotic orbit behavior of the related systems.

\section{Acknowledgement}

The work by L.Lerman was partially supported by the Laboratory of Topological Methods in Dynamics NRU HSE, of the Ministry of Science and Higher Education of RF, grant \#075-152019-1931 and by project \#0729-2020-0036. Proofs of Theorems 6,8 were performed by K.N. Trifonov under a support of the Russian Science Foundation (project 19-11-00280), Theorem 4 was proved by him under support of RFBR (grants 18-29-10081, 19-01-00607).

\section{Data availability}

The data that support the findings of this study (proofs, pictures) are placed in the body of the text. If some extra requirements appear, they should addressed to the corresponding author. 


\section{References}

[1] G.D. Birkhoff, On the periodic motions of dynamical systems, Acta math., v.50 (1927), 359-379.

[2] C.C. Conley, On the Ultimate Behavior of Orbits with Respect to an Unstable Critical Point I. Oscillating, Asymptotic, and Capture Orbits, J. Diff. Equat., v.5 (1969), 136-158.

[3] P. Duarte, Abundance of elliptic isles at conservative bifurcations, Dynam.\&Stab. Syst., v.14 (1999), No.4, 339-356.

[4] N.K. Gavrilov, L.P. Silnikov, On the three dimensional dynamical systems close to a system with a structurally unstable homoclinic curve. I. Math. USSR Sbornik 17, 467-485 (1972); II. Math. USSR Sbornik 19, 139-156 (1973).

[5] S.V. Gonchenko, L.P. Shilnikov, On two-dimensional analytic area-preserving diffeomorphisms with infiitely many stable elliptic periodic points Regular Chaotic Dyn. v.2 (1997), $106-123$.

[6] S.V. Gonchenko, L.P. Shilnikov and D.V. Turaev, Homoclinic tangencies of arbitrarily high orders in conservative and dissipative two-dimensional maps, Nonlinearity v.20 (2007), $241-275$.

[7] A.B. Katok, B. Hasselblatt. Introduction to the Modern Theory of Dynamical Systems, Cambridge Univ. Press, Revised Edition, 1993.

[8] C. Grotta Ragazzo, Irregular dynamics and homoclinic orbits to Hamiltonian saddle-centers. Commun. Pure Appl. Math., v.50 (1997), No.2, 105-147.

[9] C. Grotta Ragazzo, Nonintegrability of some Hamiltonian systems, scattering and analytic continuation, Comm. Math. Phys. v.166 (1994), 255-277.

[10] A.D. Bryuno, Normalization of a Hamiltonian system near an invariant circle or torus, Russian Math. Surv., v.44 (1989), No.2, 53-89.

[11] D. McDuff, D. Salamon. Introduction to Symplectic Topology, Second edition, Clarendon Press, Oxford, 1998.

[12] R.L. Devaney, Homoclinic orbit in Hamiltonian systems, J. Diff. Equat., v.21 (1976), 431438.

[13] R.L. Devaney. Reversible diffeomorphisms and flows. Trans. A.M.S., 218: 89-113, 1976.

[14] R.L. Devaney. Blue sky catastrophes in reversible and Hamiltonian systems. Indiana Univ. Math. J., 26: 247-263, 1977.

[15] M.S. Gonchenko, S.V. Gonchenko, On cascades of elliptic periodic points in two-dimensional symplectic maps with homoclinic tangencies, Regular and Chaotic Dynamics, 2009, Vol. 14, No. 1, 116-136.

[16] A. Delshams, M.S. Gonchenko, S.V. Gonchenko, On dynamics and bifurcations of areapreserving maps with homoclinic tangencies, Nonlinearity, v.28 (2015), 3027-3071. 
[17] A.J. Homburg, Global Aspects of Homoclinic Bifurcations of Vector Fields, Mem. Amer. Math. Soc., vol.121 (1996), no.578, 128 pp.

[18] A. Kelley, The stable, center-stable, center, center-unstable, and unstable manifolds, J. Diff. Equations, v.3 (1967), No.4, 546-570.

[19] O.Yu. Koltsova, L.M. Lerman, Periodic and homoclinic orbits in a two-parameter unfolding of a Hamiltonian system with a homoclinic orbit to a saddle-center, Int. J. Bifurcation \& Chaos, V.5 (1995), No.2, 397-408.

[20] O.Yu. Koltsova, L.M. Lerman, Families of Transverse Poincaré Homoclinic Orbits in 2NDimensional Hamiltonian Systems close to The System with a Loop to a Saddle-center, Int. J. Bifurcation \& Chaos, v.6 (1996), No.6, 991-1006.

[21] O.Yu. Koltsova, L.M. Lerman, New criterion of nonintegrability for an N-degrees-of-freedom Hamiltonian system, in "Hamiltonian Systems with three and more degrees of freedom", C.Simo, Editor, NATO ASI Series, Series C: Math. and Phys. Sciences - vol.533, Kluwer A.P., 1999, 458-470.

[22] L.M. Lerman, Hamiltonian Systems with Loops of a Separatrix of a Saddle-Center, Selecta Math. Soviet., v.10 (1991), No.3, 297-306 (transl. of a Russian paper of 1987).

[23] L.M. Lerman, Ya.L. Umanskiy, Classification of four-dimensional integrable Hamiltonian systems and Poisson actions of $\mathbb{R}^{2}$ in extended neighborhoods of simple singular points, I, Russian Acad. Sci. Sb. Math. Vol.77 (1994), No.2 511-542.

[24] L.M. Lerman, Ya.L. Umanskii, On the existence of separatrix loops in four-dimensional systems similar to the integrable Hamiltonian system, PMM USSR, Vo1.47 (1984), No.3, $335-340$.

[25] N. Kulagin, L. Lerman, A.I. Malkin, Solitons and cavitons in a nonlocal Whitham equation, Comm. Nonlin. Sci. Numer. Simul., v.93 (2021), doi.org/10.1016/j.cnsns.2020.105525.

[26] A.I. Malkin, Acoustic solitons in elastic tubes filled with a liquid, Doklady: Mechanics, v.342 (1995), No.5, 621-625 (in Russian).

[27] A. Mielke, P. Holmes, O. O'Reilly, Cascades of homoclinic orbits to, and chaos near a Hamiltonian saddle-center, J. Dyn. Different. Equat., v.4 (1992), 95-126.

[28] L. Mora, N. Romero, Persistence of homoclinic tangencies for area preserving maps, Ann. de la Faculté des Sciences de Tolouse, VI(4), 711-725.

[29] J. Moser, On the generalization of theorem of Liapounoff, Comm. Pure Appl. Math. 11:2 (1958) 257-271.

[30] J. Moser, The analytic invariants of an area-preserving mapping near a hyperbolic fixed point, Comm. Pure Appl. Math., v.9 (1956), 673-692.

[31] S. Newhouse, Diffeomorphisms with infinitely many sinks. Topology 13, 9-18 (1974)

[32] S. Newhouse, Quasi-elliptic periodic points in conservative dynamical systems. Am. J. Math. v.99 (1977), 1061-1087. 
[33] A. Poincaré. Methodes nouvelles de la mechnique celeste, v.2.

[34] H. Rüssmann, Über das Verhalten Analitischer Hamiltonscher Differential Gleichungen in der Nähe einer Gleichgewichtslösung, Math. Ann., b.154 (1964), 285-300.

[35] L.P. Shilnikov, On a Poincaré-Birkhoff problem, Math. USSR-Sb., v.3 (1967), No.3, 353-371.

[36] L.P. Sil'nikov, A contribution to the problem of the structure of an extended neighborhood of a rough equilibrium state of saddle-focus type, Math. USSR-Sbornik, v.10:1 (1970), 91-102.

[37] V.S. Afraimovich, V.V. Bykov, L.P. Shilnikov, On attractive structurally unstable sets of the Lorenz attractor type, Proc. Moscow Math. Soc., v.44 (1982), 150-212.

[38] D.V. Turaev, L.P. Shilnikov, Hamiltonian systems with homoclinic saddle curves, Dokl. Math., 39:1 (1989), 165-168.

[39] L.P. Shilnikov, S.V. Gonchenko, D.V. Turaev, Models with a structurally unstable homoclinic Poincaré curve, Dokl. Math., 44:2 (1992), 422-426.

[40] S. Smale, Diffeomorphisms with infinitely many periodic points, in "Differential and Combinatorial Topology", Ed. S Cairns. Princeton Math. Ser., Princeton, NJ: Princeton Univ. Press, 63-80.

[41] S. Smale, Differential dynamical systems, Bull. Amer. Math. Soc., v.73 (1967), No.6, 747817.

[42] D. Turaev, Hyperbolic Sets near Homoclinic Loops to a Saddle for Systems with a First Integral, Reg. Chaot. Dyn., Vol. 19 (2014), No.6, 681-693.

[43] K. Yagasaki, Horseshoes in Two-Degree-of-Freedom Hamiltonian Systems with SaddleCenters, Arch. Rational Mech. Anal. v.154 (2000), 275-296. 